\title{
Lysosomal Exocytosis Releases Pathogenic a-Synuclein Species from Neurons
}

Ying Xue Xie ${ }^{1 \#}$, Nima N. Naseri2\#, Jasmine Fels ${ }^{1}$, Parinati Kharel ${ }^{1}$, Yoonmi Na ${ }^{1}$, Jacqueline Burré $^{1}$, Manu Sharma ${ }^{1 *}$

1 Appel Institute for Alzheimer's Research, and Brain \& Mind Research Institute, Weill Cornell Medicine, New York, NY, USA.

2 Department of Chemistry, University of Pennsylvania, Philadelphia, PA, USA.

\# Equal contribution

* Correspondence: mas2189@med.cornell.edu 


\section{SUMMARY}

Considerable evidence supports the release of pathogenic aggregates of the neuronal protein $\alpha$-Synuclein (aSyn) into the extracellular space. While this release is proposed to instigate the neuron-to-neuron transmission and spread of aSyn pathology in synucleinopathies including Parkinson's disease, the molecular-cellular mechanism(s) remain unclear. Here we show that pathogenic species of aSyn accumulate within neuronal lysosomes in mouse brains and primary neurons. We then find that neurons release these pathogenic aSyn species via SNAREdependent lysosomal exocytosis; proposing a central mechanism for exocytosis of aggregated and degradationresistant proteins from neurons.

\section{INTRODUCTION}

Cytoplasmic aggregates of the synaptic protein aSyn are a characteristic feature of multiple neurodegenerative diseases termed "synucleinopathies", including Parkinson disease (PD). Over the course of these age-linked diseases, aSyn assembles into amyloid-type $\beta$-sheet rich aggregates, depositing as Lewy bodies and/or Lewy neurites within neurons (Baba et al., 1998; Spillantini et al., 1998; Spillantini et al., 1997). The neuro-anatomical propagation of $\alpha$ Syn pathology is highly stereotyped, and has been defined well-enough to be used in staging PD - originating in the medulla and olfactory bulb, then advancing to the midbrain and basal forebrain, followed by "spread" to the neocortex (Braak et al., 2002; Braak et al., 2003). This observation, combined with detection of extracellular aSyn in human cerebrospinal fluid (El-Agnaf et al., 2003), led to the original conjecture that pathogenic species of aSyn may be transmitted neuron-to-neuron via prion-like permissive templating. Subsequently, in PD patients who had received fetal neuron transplants, appearance of Lewy pathology in the undiseased grafted neurons also pointed to the transmission of aSyn pathology from the surrounding PDaffected neurons (Kordower et al., 2008; Li et al., 2008). These observations were followed by animal studies which confirmed either neuron-to-neuron transmission of aSyn pathology (Desplats et al., 2009), or induction of intraneuronal aSyn pathology by an inoculum of extracellular aSyn fibrils (Luk et al., 2012; Volpicelli-Daley et al., 2011a).

However, unclarity persists in how the cytosolic aggregates are conveyed into the extracellular milieu: Proposed pathway(s) include secretion of aSyn monomers, oligomers and/or larger aggregates inside extracellular vesicles - such as exosomes (Danzer et al., 2012; Emmanouilidou et al., 2010) or microvesicles (Minakaki et al., 2018) - which have been categorized collectively as "unconventional secretion". Other proposed mechanisms include synaptic vesicle release (Yamada and Iwatsubo, 2018), or exocytosis of other types of vesicles (Jang et al., 2010; Lee et al., 2005), exophagy (Ejlerskov et al., 2013), membrane translocation (Ahn et al., 2006), or trafficking of aggregates through tunneling nanotubes (Abounit et al., 2016). Yet, all of these studies have not identified a neuronal organelle that accumulates and then secretes the pathogenic aSyn aggregates, resulting in the current mechanistic gap.

Here, we present our finding that pathogenic aSyn species accumulate within the neuronal lysosomes in mouse brains and in primary neurons, and that these species are released from neurons via SNARE-dependent lysosomal exocytosis. 


\section{RESULTS}

\section{Pathologenic species of aSyn accumulate within the lysosomes of transgenic $\alpha S^{2}{ }^{A 53 T}$ mouse brains}

Rapid neurodegeneration and synapse loss in cysteine string protein- $\alpha$ knockout mice $\left(\mathrm{CSPa}^{-/}\right)$is rescued by transgenic expression of aSyn carrying the PD mutation A53T (Tg- $\alpha S^{A 5}{ }^{A 53 T}$ ) (Chandra et al., 2005). However, beyond 5 months, the rescued Tg- $\alpha \mathrm{Syn}^{\mathrm{A}}{ }^{\mathrm{5} 3 \mathrm{~T}} / \mathrm{CSP}^{-/-}$mice begin exhibiting neurodegeneration due to the transgenic overexpression of $\alpha \mathrm{Syn}^{\mathrm{A}}{ }^{\mathrm{A53T}}$. Curiously, we found that $\mathrm{Tg}-\alpha \mathrm{Syn}^{\mathrm{A} 53 \mathrm{~T}} / \mathrm{CSPa}^{-/-}$mice have accelerated accumulation of the pathogenic versions of $\alpha \mathrm{Syn}$ compared to Tg- $\alpha \mathrm{Syn}^{\mathrm{A5} 3 \mathrm{~T}} / \mathrm{CSPa}^{+/-}$mice, detectable by phospho-Ser129 specific, filamentous $\alpha$ Syn-specific, and amyloid-specific antibodies (Supplementary Fig. S1). CSPa ${ }^{+/-}$mice have normal CSPa function (Fernández-Chacón et al., 2004), and there

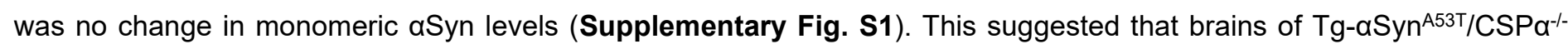
mice are collecting aSyn aggregates faster in the absence of CSPa.

Loss-of-function mutations in CSPa cause the lysosomal storage disorder adult-onset neuronal ceroid lipofuscinosis (ANCL) or Kufs disease (Benitez et al., 2011; Cadieux-Dion et al., 2013; Nosková et al., 2011; Velinov et al., 2012). Specifically, loss of CSPa function leads to progressive accumulation of lysosomes containing undegraded material such as lipofuscin/residual bodies, in ANCL patient brains (Benitez et al., 2011), in CSP ${ }^{-1-}$ mouse brains (Fernández-Chacón et al., 2004), in Drosophila models carrying the ANCL mutations (Imler et al., 2019), and in ANCL patient-derived induced neurons

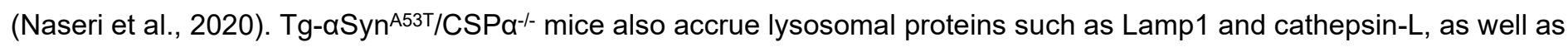
ATP5G - a mitochondrial protein characteristically stored in lysosomes of CSPa loss-of-function models and ANCL patient

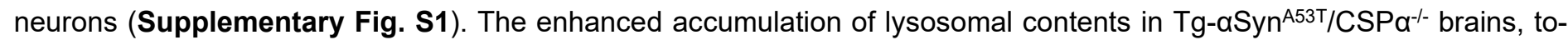
gether with the exaggerated buildup of aSyn aggregates in these brains gave us the clue that aSyn aggregates may be depositing in the lysosomes.

We next aimed to determine whether $\alpha$ Syn aggregates accumulate within lysosomes, even in the absence of CSPaI--driven lysosomal pathology. Thus, we tested whether aged Tg- $\alpha S_{4 n}{ }^{A 53 T}$ mice, which display severe $\alpha$ Syn-driven pathology, have aSyn aggregates within their lysosomes.

We found pathogenic aSyn species, but not monomeric aSyn, in dextranosomes (heavy lysosomes loaded with dextran to enhance purity) isolated from the brains of 6 month old Tg- $\alpha S_{y n}{ }^{\mathrm{A} 53 \mathrm{~T}}$ mice (Fig. 1 a-c). These lysosomal fractions precisely coincided with pathogenic aSyn species, and had highly enriched lysosomal proteins as well as cathepsin-D activity, with negligible contamination from other organelle protein-markers or mitochondrial and peroxisomal enzyme activities (Fig. 1 a-c). This experiment indicates that isolated intact lysosomes from aged Tg- $\alpha \mathrm{Syn}^{\mathrm{A53T}}$ mice contain pathogenic $\alpha \mathrm{Syn}$ species.

\section{Pathogenic species of aSyn accumulate within the lumen of neuronal lysosomes}

The lysosomes studied above are a mixture of lysosomes from all brain cells. To isolate and study lysosomes specifically from neurons, we generated transgenic mice expressing ${ }^{\mathrm{HA}}$ Lamp $1^{\text {Myc }}$ - lysosomal protein Lamp1 epitope-tagged 
on its luminal domain with tandem $2 x H A$, and on its cytoplasmic tail with 2xmyc - driven by the neuron-specific synapsin-I promoter (Supplementary Fig. S2 a-d), enabling affinity-isolation of neuronal lysosomes from mouse brains. The Tg-

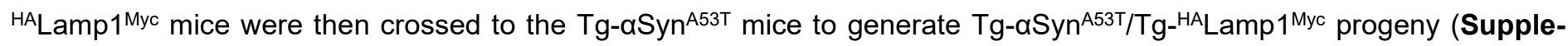
mentary Fig. S3 a-g). Immuno-isolation experiments from aged Tg- $\alpha S_{S y}{ }^{A 53 T / T g-H A L a m p 1}{ }^{\text {Myc }}$ mouse brains indicated that pathogenic versions of aSyn co-isolate with neuronal lysosomes (Fig 2a). We found that $\sim 10 \%$ of the SDS-resistant aSyn aggregates, and $\sim 20 \%$ of the more mature aSyn aggregates (filamentous and amyloid-type) reside within neuronal lysosomes (Fig. 2a). This result indicates that a non-trivial portion of pathogenic aSyn species in the brain are found in neuronal lysosomes.

To understand the fate of these lysosomal aSyn aggregates, we developed a neuronal model which accumulates

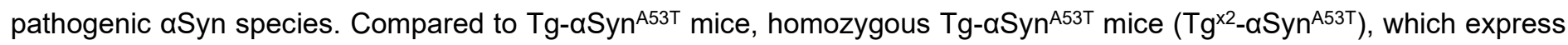
twice the amount of transgenic $\alpha S_{y}{ }^{A 53 T}$, have shorter lifespans, earlier onset of neuromuscular pathology, and begin accumulating pathogenic aSyn species earlier - by only 6 weeks of age (Suppl. Fig. S4 a-f) - which is achievable in longlived primary neuron cultures. Accordingly, primary neurons from Tg ${ }^{\times 2}-\alpha S^{A}{ }^{A 53 T}$ mice also accrued pathogenic $\alpha$ Syn species by 6 weeks, including aggregates detectable by antibodies against aSyn fibrils and amyloid-type conformations (Suppl Fig. S4g).

To specifically label and track the pathogenic aSyn species located within neuronal lysosomes, we targeted Apex2 to the lysosomal lumens of $\mathrm{Tg}^{\mathrm{\times 2}-\alpha S y n}{ }^{\mathrm{A53T}}$ primary neurons. We lentivirally expressed a synapsin-1 promoter-driven construct, comprised of Apex-2 fused to the luminal domain of a truncated version of Lamp1 (Apex-2Lamp1) (Suppl Fig. S5 a-c), which proximity-labels the luminal contents of neuronal lysosomes with biotin. In these $\mathrm{Tg}^{\times 2}{ }^{\alpha{ }^{2}} \mathrm{Syn}^{\mathrm{A53T}}$ neurons expressing Apex-2Lamp1, we found that pathogenic $\alpha$ Syn species as well as lysosomal proteins (typified by cathepsin-L) were biotinylated (Fig. 2b), confirming that pathogenic aSyn species accumulate within the lumen of neuronal lysosomes.

\section{Pathogenic aSyn species are secreted from neurons via SNARE-dependent lysosomal exocytosis}

Release of aSyn from neurons, particularly the seeding-competent pathogenic versions of aSyn, is considered a key step in the spatial progression of synucleinopathies (Peng et al., 2020), and aSyn has been documented in extracellular fluids of human PD patients (El-Agnaf et al., 2003). We also found pathogenic species of aSyn in the cerebrospinal fluid of aged Tg-aSyn ${ }^{A 53 T}$ mice (Suppl. Fig. S6). Importantly, this extracellular pool of aSyn species appears not to be enveloped in membranes, as they were not protected from proteinase K digestion (Suppl. Fig. S6). Proteolytic susceptibility was also unaffected by the presence of detergent, akin to the lysosomal luminal protein cathepsin- $L$ and the constitutively secreted neuronal protein neuroserpin (Suppl. Fig. S6). This was contrary to the well-protected contents of exosomes, typified by TSG101, which becomes susceptible to proteolysis only in the presence of detergent (Suppl. Fig. S6). Accumulation inside lysosomes of the pathogenic aSyn species, together with their un-enveloped presence in the extracellular space, prompted us to investigate whether lysosomes are releasing aSyn aggregates contained inside them.

We thus collected the medium from $\mathrm{Tg}^{\times 2}-\alpha \mathrm{Syn}^{\mathrm{A53T}}$ primary neurons, in which the lysosomal contents had been biotinylated via neuron-specific expression of Apex-2Lamp1 (as in Fig. 2b and Suppl. Fig. S5). We found that lysosomal contents, typified by cathepsin-L, as well as pathogenic aSyn species could be precipitated from the medium via their biotin- 
modification (Fig. 3a). In contrast, exosomal contents, typified by TSG101, were not biotinylated/precipitated. This result points to lysosomal exocytosis - SNARE-dependent fusion of lysosomes with the plasma membrane - as the likely mechanism for the exit of aSyn aggregates from neurons.

To test this hypothesis, we modulated the SNARE proteins responsible for lysosome-to-plasma membrane fusion. First, we found VAMP7 as the prominent v-SNARE in immunoisolated lysosomes (Suppl. Fig. S7a). We then screened for the Qb-type t-SNAREs which interact and thus co-immunoprecipitate with VAMP7. SNAP23 was the clearest Qb/t-SNARE candidate (Suppl. Fig. S7a). Based on these results and prior studies validating them (Rao et al., 2004), we tested four strategies to disrupt lysosomal exocytosis: shRNA knockdown of either SNAP23 (SNAP23 ${ }^{\mathrm{KD}}$ ) or VAMP7 (VAMP7 ${ }^{\mathrm{KD}}$ ), and overexpression of dominant negative fragments of either SNAP23 (SNAP23 ${ }^{\mathrm{DN}}$ ) or VAMP7 (VAMP7 ${ }^{\mathrm{DN}}$ ). In wild type primary neurons, the release of cathepsin-L was used to signal lysosomal exocytosis, opposed to the constitutively secreted neuroserpin. VAMP7KD and SNAP23DN had no significant effect on lysosomal exocytosis, but VAMP7DN severely reduced it by $\sim 80 \%$ and SNAP23KD partially reduced it by $\sim 40 \%$ (Suppl. Fig. S7 b-c). Therefore, we applied the latter two strategies to disrupt lysosomal exocytosis next.

Importantly, in $\mathrm{Tg}^{\times 2}$-aSyn ${ }^{\mathrm{A53T}}$ neurons, both VAMP7 ${ }^{\mathrm{DN}}$ and SNAP23 ${ }^{\mathrm{KD}}$ strategies reduced the release of pathogenic aSyn species into the medium, and the effect of SNAP23 ${ }^{\mathrm{KD}}$ was rescued by overexpression of a knockdown-resistant version of wild type SNAP23 (Fig. 3b). Moreover, the release of pathogenic $\alpha$ Syn species was significantly correlated with the change in lysosomal exocytosis, measured as cathepsin-L released into the medium (Fig. $\mathbf{3} \mathbf{b}$-c). These results suggest that SNARE-dependent exocytosis of lysosomes is essential and rate-determining for the release of pathogenic aSyn species from neurons.

\section{aSyn species released via lysosomal exocytosis can seed aggregation of purified recombinant aSyn}

Pathogenic spread requires that the released aSyn is able to template or "seed" the assembly of amyloid-type aggregates from monomeric aSyn. To test whether the pathogenic aSyn species exocytosed from neurons are seedingcompetent, we performed in vitro aggregation of purified recombinant myc-tagged wild type aSyn (Suppl. Fig. S8), in the presence of extracellular media collected from neuron cultures generated either from wild type mice, or from $\operatorname{Tg}^{\times 2}-\alpha S^{2} n^{A 53 T}$ mice with or without lentiviral expression of VAMP7DN fragment (Fig. 4 a-c). Aggregation kinetics, measured by amyloidbinding fluorescent dye K114 (Fig. 4a) or Thioflavin-T (Fig. 4b), were enhanced by medium from Tg $^{\times 2}$ - $\alpha S^{2} y n^{A 53 T}$ neurons, compared to the medium from wild type neurons. Suppressing lysosomal exocytosis in the $\mathrm{Tg}^{\times 2}-\alpha \mathrm{Syn}^{\mathrm{A5} 3 \mathrm{~T}}$ neurons, via VAMP7DN expression, diminished the aggregation-promoting effect of the $\mathrm{Tg}^{\times 2}$ - $\alpha \mathrm{Syn}^{\mathrm{A} 53 \mathrm{~T}}$ medium (Fig. 4 a-b). When mycaSyn aggregation kinetics were measured by quantitative immunoblotting for either disappearance of aSyn monomers or for the appearance of filamentous and amyloid-type aggregates, we again found that compared to the wild type neuron medium, aggregation was augmented by the $\mathrm{Tg}^{\times 2}-\alpha \mathrm{Syn}^{\mathrm{A} 53 \mathrm{~T}}$ medium, and the expression of VAMP7DN fragment diminished this effect (Fig. 4c). In all the assays, a lag-time of nearly 2 weeks is apparent before the appearance of amyloid signal with the wild type medium (Fig. 4a-c). Importantly, this lag is shortened or eliminated by the aggregates in $\mathrm{Tg}^{\times 2}$ - $\alpha \mathrm{Syn}^{\mathrm{A53T}} \mathrm{medium}^{\mathrm{A}}$ (Fig. 4a-C), with a reduced effect when lysosomal exocytosis is suppressed by VAMP7DN fragment (Fig. 4a-c). This seeding activity of the lysosomally released aSyn species indicates that they are capable of amyloid-nucleation via permissive templating. 


\section{DISCUSSION}

Release of aSyn aggregates from neurons is thought to transmit neuropathology to other yet-unaffected neurons. Here we find that pathogenic aSyn species accumulate within neuronal lysosomes in mouse brains and in primary neurons, and are then released from neurons via SNARE-dependent lysosomal exocytosis.

It is important to note that for aSyn pathology to transmit from neuron-to-neuron, the lysosomal exocytosis mechanism is more in line with the following observations, which underline that the extracellular pool of pathogenic aSyn is likely not membrane-enveloped as in exosomes, extracellular vesicles, or nanotubes, but is non-enveloped: a) the stark pathology and rapid propagation caused by inoculated pre-formed fibrils (Luk et al., 2012; Luk et al., 2009; Paumier et al., 2015; Rey et al., 2018; Rey et al., 2013; Volpicelli-Daley et al., 2011b), b) the efficacy of humoral and cellular immunotherapy against aSyn (reviewed in (Chatterjee and Kordower, 2019)), c) the efficacy of passive immunization using antibodies against aSyn epitopes (reviewed in (Chatterjee and Kordower, 2019)), and d) much of the extracellular aSyn has been found as nonenveloped (Borghi et al., 2000; El-Agnaf et al., 2003; Sung et al., 2005), while a minority of aSyn is contained in extracellular vesicles or exosomes (Ejlerskov et al., 2013; Emmanouilidou et al., 2010; Jang et al., 2010).

Key gaps still remain in how the pathogenic aSyn species are targeted and trafficked to lysosomes, as well as in the fate of extra cellular aSyn aggregates following their release from neurons:

The aggregates/fibrils of aSyn could form inside the acidified lumen of lysosomes (Buell et al., 2014), either from smaller oligomers or monomers, especially if their degradation is delayed due to reduced/disrupted lysosomal activity (Malik et al., 2019). Yet, we found no presence of aSyn monomers within isolated lysosomes, making this possibility less likely. In contrast, macro-autophagy of ready-formed aggregates from the cytoplasm, termed "aggrephagy", is a more likely mechanism, following from the well-recorded ability of autophagosomes to encapsulate large cytoplasmic structures including aggregates (Yamamoto and Simonsen, 2011), and target them to lysosomes.

Once released from the neurons, the fate of extracellular/interstitial $\alpha$ Syn aggregates also remains unclear, and can follow multiple possible scenarios: uptake into microglia (Lee et al., 2008), astrocytes (Loria et al., 2017), and/or oligodendrocytes (Reyes et al., 2014), and/or drainage via glymphatics (Rasmussen et al., 2018; Smith and Verkman, 2018) or blood-flow (El-Agnaf et al., 2003; Foulds et al., 2013). However, for the extracellular aSyn aggregates to cause the stereotypic "spread" of pathology via a prion-like etiology, other neurons will have to take up the released aggregates, and this process remains even less understood than the glial uptake or clearance scenarios.

The results presented here provide evidence of the accumulation of pathogenic aSyn species in neuronal lysosomes, and pinpoint to lysosomal exocytosis as a pathway of release for these pathogenic aSyn species into the extracellular milieu. This pathway, at first sight, appears to have relevance to the release of undegraded aggregates composed of other proteins as well that transmit pathology from cell-to-cell (reviewed in (Peng et al., 2020)), and follow-up studies will be needed to test this speculation. 


\section{FIGURES \& LEGENDS}

a

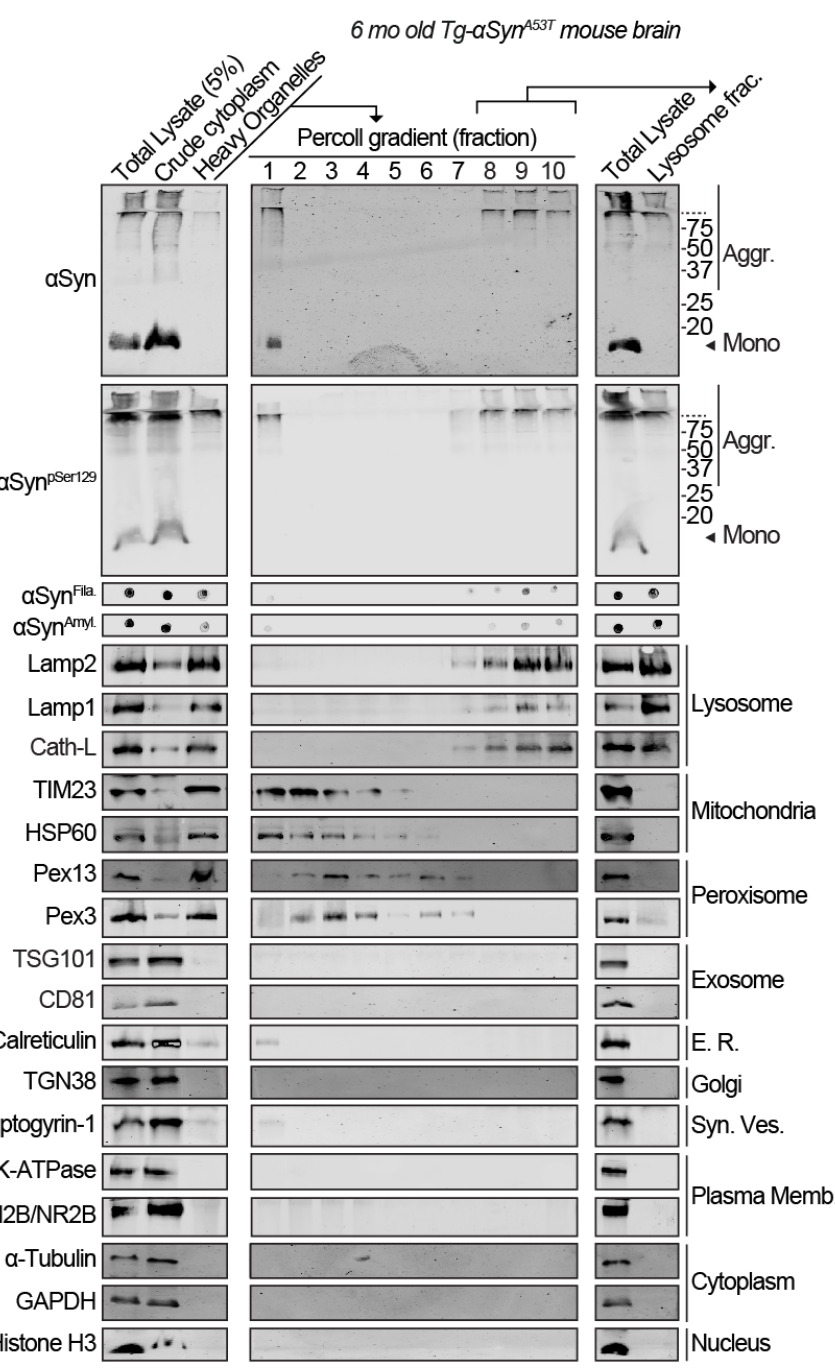

C
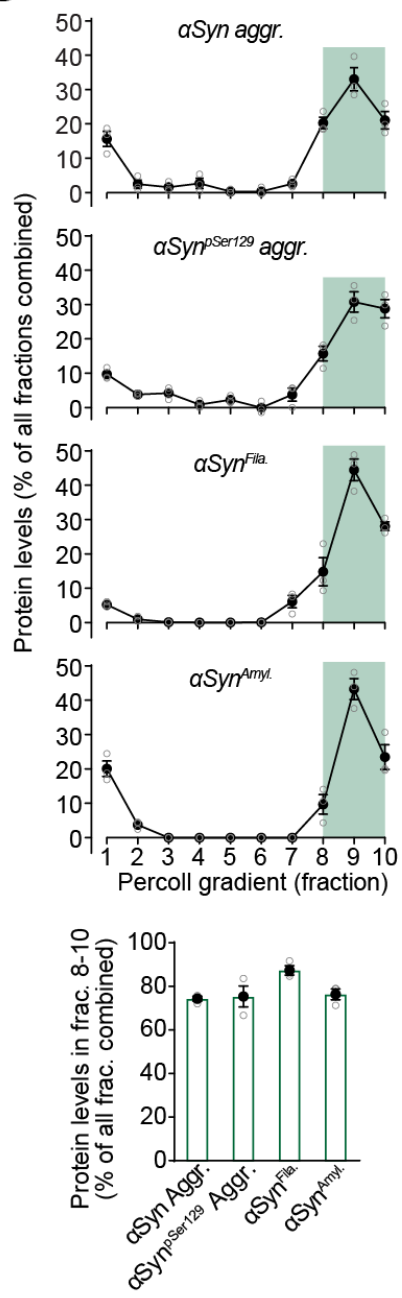

b
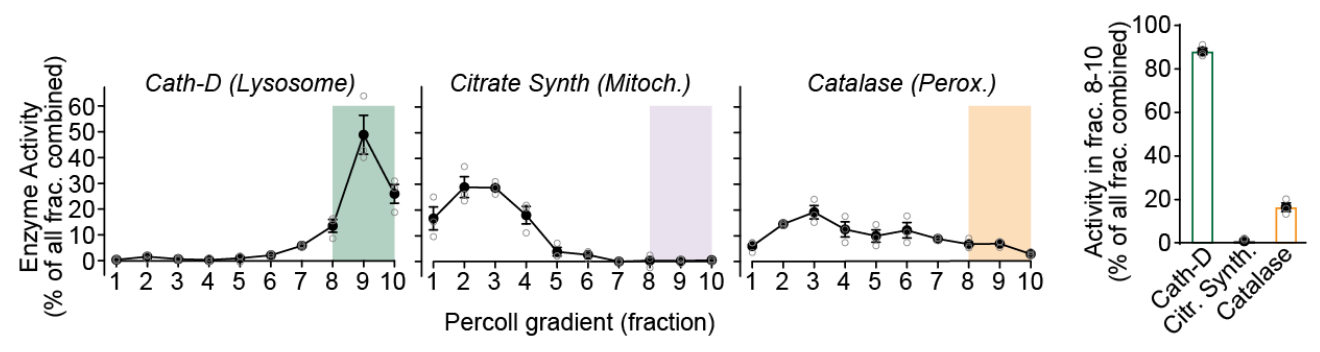

Figure 1 | Pathogenic aSyn aggregates accumulate within lysosomes of aged Tg- $\alpha \mathrm{Syn}^{\mathrm{A53T}}$ mice.

Lysosomes were isolated from 6 month old Tg- $\alpha S^{S y n}{ }^{A 53 T}$ mouse brains via Percoll gradient centrifugation of the heavy organelle fraction - which contained peroxisomes, heavy lysosomes loaded in vivo with dextran-70, and mitochondria swollen ex vivo by $\mathrm{CaCl}_{2}$. (a) Lysosome (dextranosome) enrichment was determined by immunoblotting for markers of indicated organelles, compared to the respective levels in the total lysate input. (b) Left panels - Activities of enzymes contained within lysosomes (cathepsin-D), mitochondria (citrate synthase), and peroxisomes (catalase) were measured, testing for isolation of intact organelles. Right panel - Summary graph of enzyme activity present in the combined "lysosomal 
fractions" (fractions 8-10). (c) Top panels - Levels in each Percoll gradient fraction of pathogenic aSyn species: aggregated (aSyn Aggr), aggregates phosphorylated at Ser129 (aSyn ${ }^{\mathrm{pSer} 129}$ Aggr), amyloid-type ( $\alpha$ Syn ${ }^{\text {Amyl) }}$, and filamentous $\left(\alpha S y n^{\text {Fila }}\right.$ ). Bottom panel - Summary graph of these aSyn species present in the combined "lysosomal fractions" (fractions 8-10). ( $n=3)$. (b-c) All data in are shown as means \pm SEM, where ' $n$ ' represents mouse brains. 
a
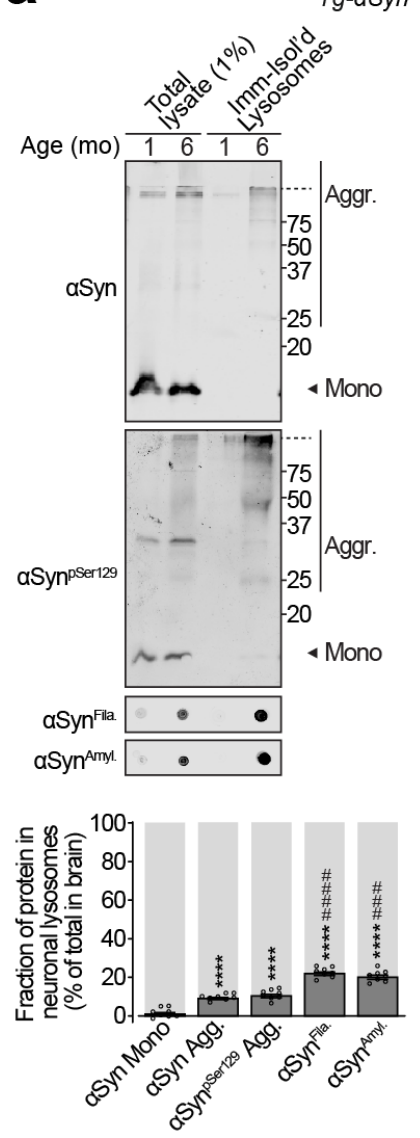

b

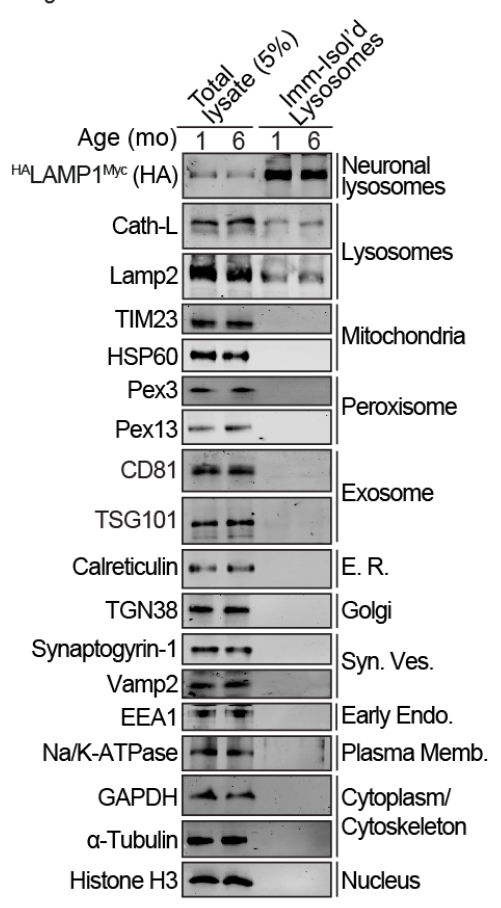

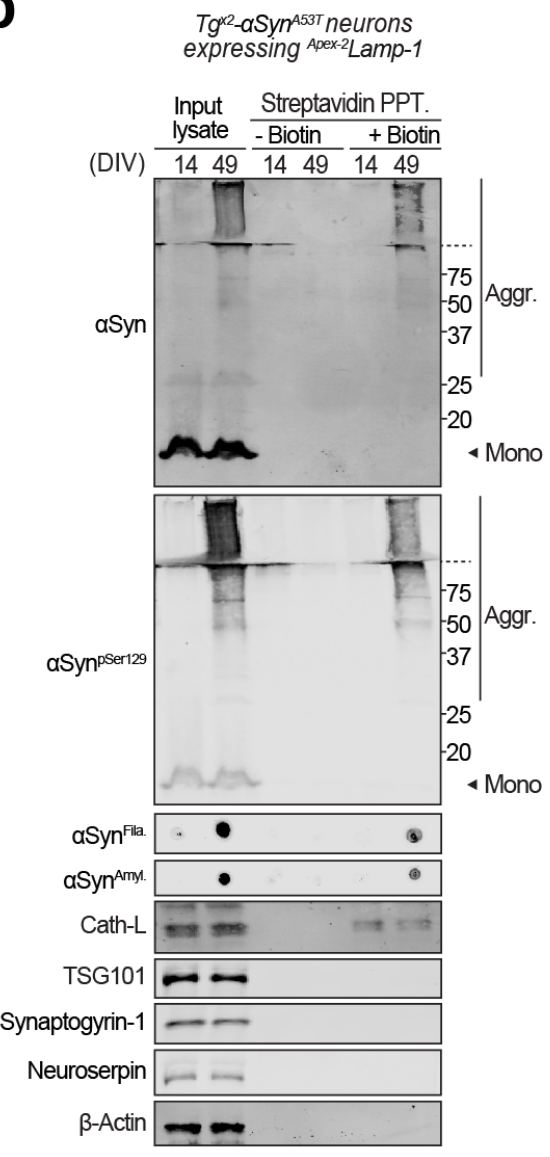

Figure 2 | Pathogenic aSyn species accumulate within neuronal lysosomes in mouse brains and primary neurons.

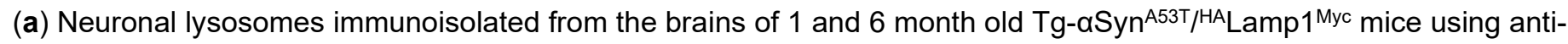
myc antibody were analyzed by immunoblotting for levels of $\alpha$ Syn, $\alpha \operatorname{Syn}^{\mathrm{pSer} 129}, \alpha-\mathrm{Syn}^{\mathrm{Fila}}$, and $\alpha \mathrm{Syn}^{\mathrm{Amyl}}$ (Mono = monomer, Aggr $=$ aggregates). Fraction of these aSyn species residing within the lysosomes at 6 months of age (bottom graph); this was back-calculated from the fraction of each aSyn species immuno-captured, normalized to the fraction of neuronal lysosomes captured - indicated by the fraction of ${ }^{H A}$ Lamp1 ${ }^{\text {Myc }}$ captured. Immunoblots against markers of lysosomes and of other organelles are also shown $(n=8)$. (b) $\operatorname{Tg}^{\times 2}-\alpha S{ }^{A 53 T}$ primary cultures lentivirally expressing a synapsin-1 promoterdriven Apex-2Lamp1 construct were subjected to proximity-labeling with biotin at 14 or 49 days in vitro (DIV). Labeled proteins were precipitated on streptavidin-coated magnetic beads and immunoblotted for the indicated pathogenic aSyn species, as well as for marker proteins cathepsin-L (lysosomes), TSG101 (exosomes), synaptogyrin-1 (synaptic vesicles), neuroserpin (constitutively secreted neuronal protein), and $\beta$-actin (cytosol) (representative of $n=3$ ). Data represent means \pm SEM. Each ' $n$ ' corresponds to independently aged mouse littermates in (a) and independent primary neuron cultures in

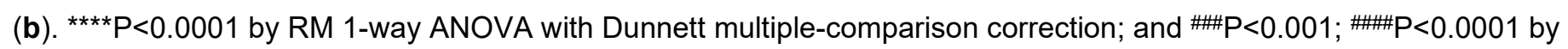
non-parametric Friedman test with Dunn's multiple-comparison adjustment. 

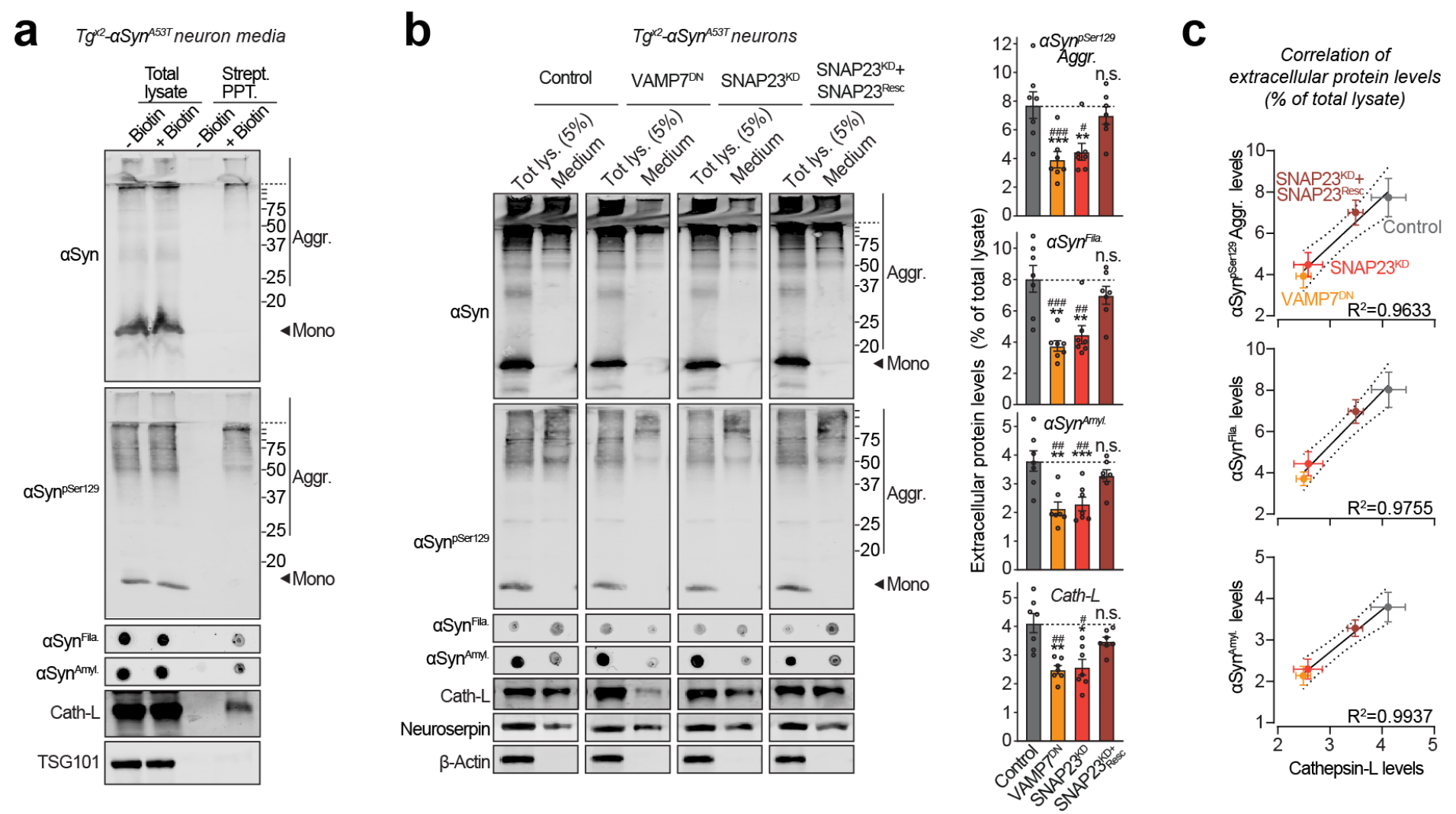

Figure 3 | Pathogenic aSyn species are secreted from neurons via SNARE-dependent lysosomal exocytosis.

(a) $\mathrm{Tg}^{\times 2}-\alpha \mathrm{Syn}^{\mathrm{A} 53 \mathrm{~T}}$ primary neurons lentivirally expressing a synapsin-1 promoter-driven Apex-2Lamp1 (infected on DIV 7) were subjected to proximity-labeling on DIV 47. Biotinylated proteins released into the media during $48 \mathrm{~h}$ (by DIV 49) were

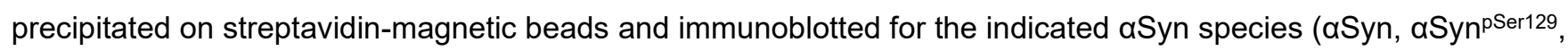
$\alpha S_{y}{ }^{A m y l}$, and $\left.\alpha S y n^{F i l a}\right)$, lysosome luminal protein cathepsin-L, and exosome luminal protein TSG101. Strept. PPT. = streptavidin precipitate. (representative of $n=3$ ). (b) Media collected over 2 days (DIV 47-49) from Tg $^{\times 2}-\alpha{ }^{2}{ }^{A 53 T}$ primary neurons lentivirally expressing control (GFP), GFP-VAMP7 ${ }^{\mathrm{DN}}$ fragment, SNAP23 ${ }^{\mathrm{KD}}$ shRNA, and SNAP23 ${ }^{\mathrm{KD}}$ shRNA plus knockdown-resistant SNAP23 rescue-construct (infected on DIV 7), were immunoblotted for the indicated aSyn species ( $\alpha$ Syn, $\alpha S_{y n}{ }^{p S e r 129}, \alpha S y n^{A m y l}$, and $\alpha S y n^{\text {Fila }}$ ), cathepsin-L (lysosome luminal), neuroserpin (constitutively secreted from neurons), and $\beta$-actin (cytoplasmic). For quantification (graphs on the right) protein level in medium was normalized to its levels in total cellular lysate $(5 \%$ loaded) $(n=7)$. (c) Correlation between the levels of pathogenic aSyn species and the levels of cathepsin-L released in the medium, upon the indicated manipulation of SNARE-dependent lysosomal exocytosis; derived from data shown above in panel (b). Linear regression is shown with dotted lines depicting the $95 \%$ confidence intervals, and Pearson's correlation coefficient is indicated on the bottom right of each graph. $(n=7)$. All data represent means \pm SEM. Each ' $n$ ' corresponds to a separate mouse litter used for a batch of neuronal culture and infection. In (b) * $P<0.05$; ${ }^{* *} \mathrm{P}<0.01$; ${ }^{* * *} \mathrm{P}<0.001$; by RM 1-way ANOVA with Dunnett multiple-comparison correction; and ${ }^{\#} \mathrm{P}<0.05$; ${ }^{\# \#} \mathrm{P}<0.01$; ${ }^{\#} \mathrm{P}<0.001$ by non-parametric Friedman test with Dunn's multiple-comparison adjustment. 
a

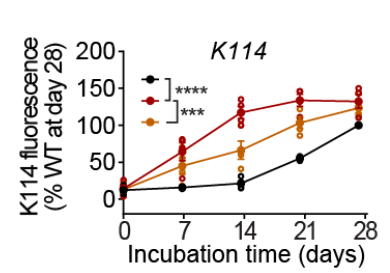

b

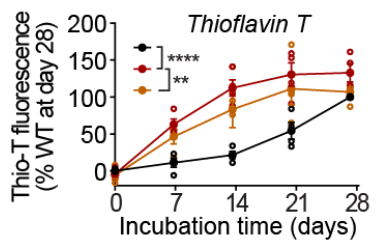

$\rightarrow$ WT

$\rightarrow-\operatorname{Tg}^{\mathrm{X}}-\alpha \mathrm{S} y n^{\mathrm{A} 53 \mathrm{~T}}$

$\rightarrow-\mathrm{Tg}^{\mathrm{x}-}-\mathrm{aSyn} \mathrm{n}^{\mathrm{A} 3 \mathrm{~T}}+\mathrm{VAMP7} 7^{\mathrm{DN}}$

C
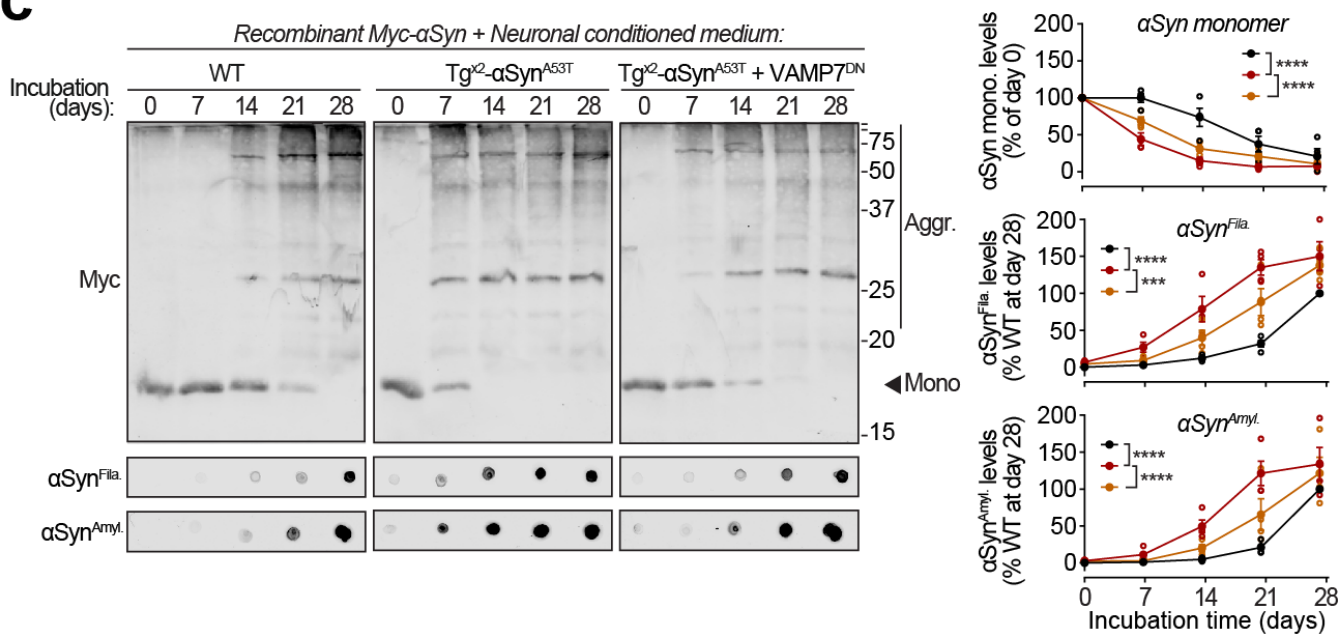

Figure 4 | Seeding of recombinant $\alpha$ Syn aggregation by pathogenic aSyn species exocytosed from neurons.

(a-c) Recombinant purified myc-aSyn was shaken at $37^{\circ} \mathrm{C}$ in presence of concentrated extracellular medium from mouse cortical neuron cultures collected over 7 days (DIV 42-49), generated either from wild type (WT) mice, or from Tg²aSyn ${ }^{\mathrm{A53T}}$ mice with or without lentiviral expression of VAMP7 dominant-negative fragment (VAMP7DN; infected at DIV 7). Aggregation of myc-aSyn was analyzed at the indicated days of incubation by the following assays: (a) Congo-red derivative, amyloid-binding dye K114 fluorescence at 390/535 nm (n=4). (b) Amyloid-binding dye Thioflavin-T fluorescence at $450 / 485 \mathrm{~nm}(\mathrm{n}=4)$. (c) Quantitative immunoblotting for the myc epitope-tag, where aggregation is measured as disappearance of monomeric myc- $\alpha$ Syn (top; $n=4$ ); dot-blotting for filamentous myc- $\alpha$ Syn aggregates using $\alpha$ Syn $n^{\text {Fila }}$ antibody (middle; $n=4$ ); and dot-blotting for amyloid-type myc- $\alpha$ Syn aggregates using $\alpha$ Syn ${ }^{\text {Amyl }}$ A11 antibody (bottom; $n=4$ ). All data represent means \pm SEM, where each ' $n$ ' is an independent aggregation experiment. ${ }^{*} P<0.05$; ${ }^{* *} P<0.01$; ${ }^{* * *} P<0.001$; 

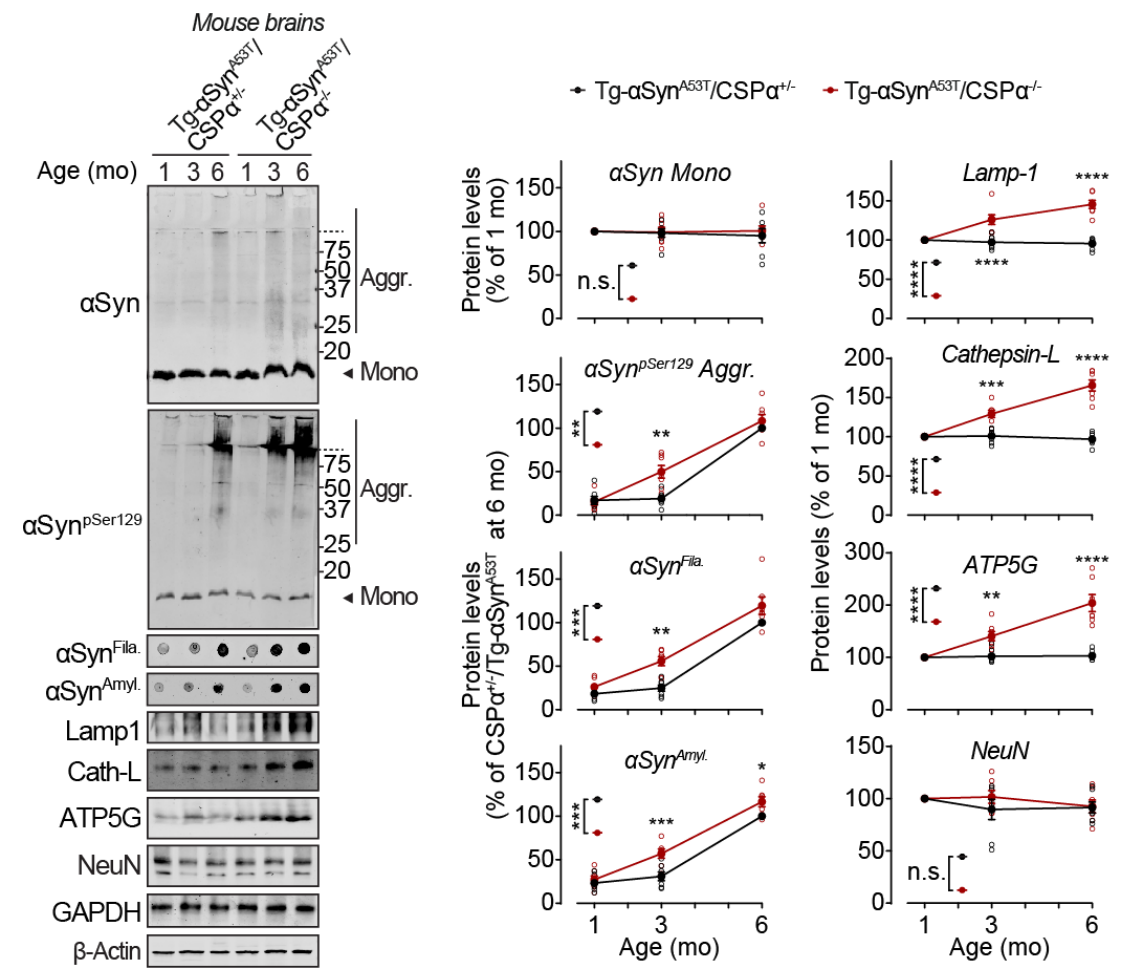

\section{Supplementary Figure S1 | Accelerated accumulation of pathogenic aSyn aggregates and lysosomal proteins in} Tg-aSyn ${ }^{A 53 T}$ mouse brains due to the loss of CSPa function.

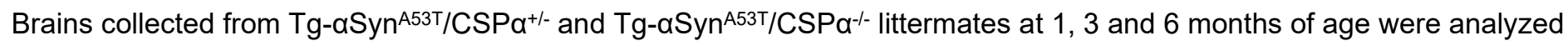
by quantitative immunoblotting for the following versions of aSyn: monomeric (aSyn Mono), phosphorylated at Ser129

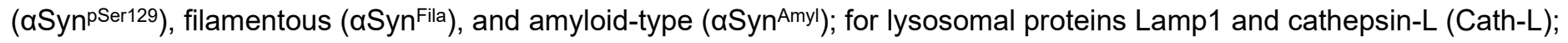
for ATP5G, which accumulates in lysosomes storage caused by loss of CSPa function; as well as for neuronal marker NeuN. These were all normalized to $\beta$-actin levels. Mono = monomer; Aggr. = aggregates. $(n=7)$

All data are shown as means \pm SEM, where ' $n$ ' represents littermate mouse brains. ${ }^{*} P<0.05$; ${ }^{* *} P<0.01$; ${ }^{* * *} P<0.001$; ${ }^{* * * *} \mathrm{P}<0.0001$ by RM 2-way ANOVA with Bonferroni multiple comparisons post-test. 
a
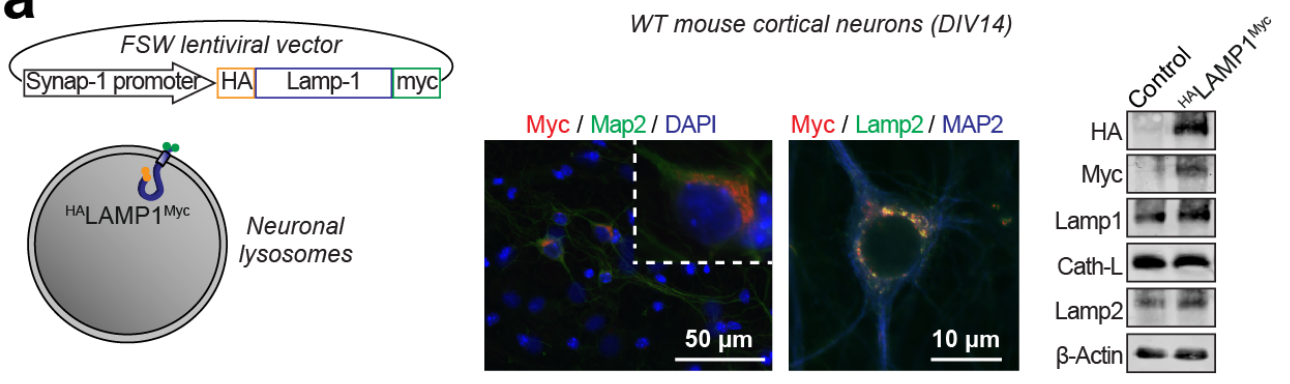

$10 \quad \operatorname{Tg}^{H A} L A M P 1^{M y c}$ mouse generation
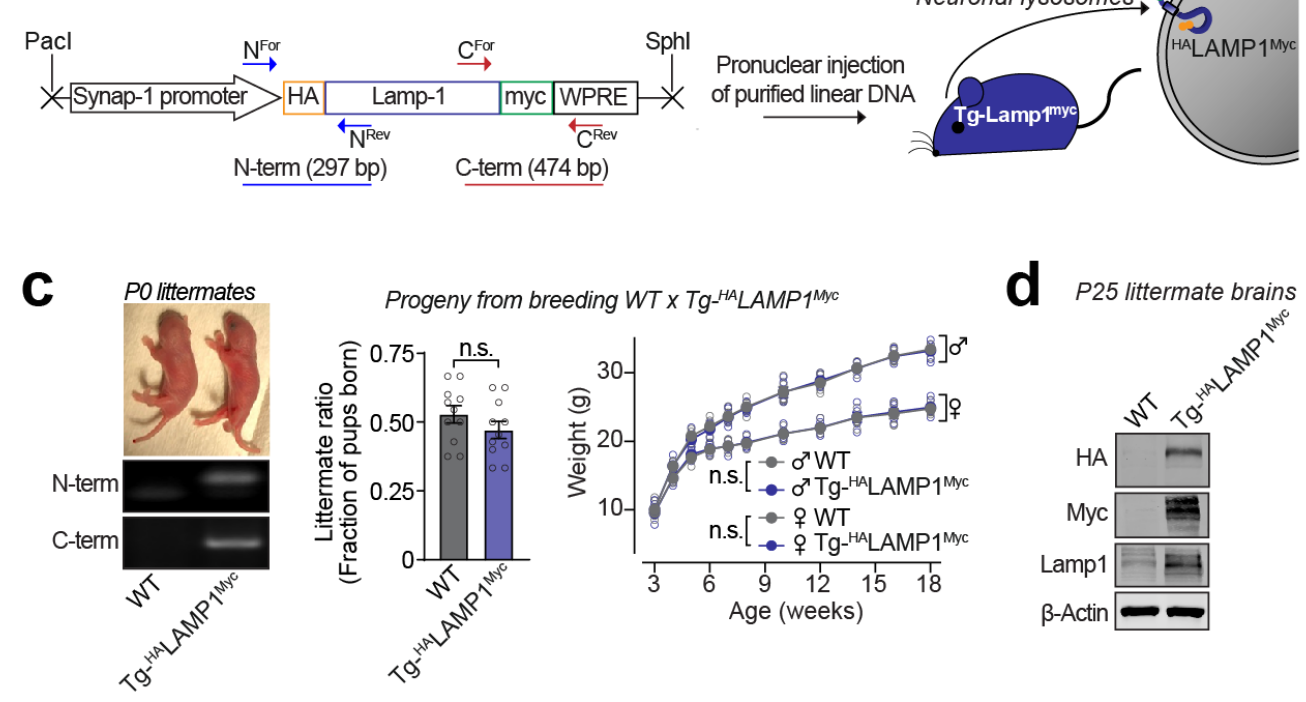

Supplementary Figure S2 | Development of a transgenic mouse model to isolate neuronal lysosomes from brains.

(a) ${ }^{H A}$ Lamp1 ${ }^{\text {Myc }}$ construct lentivirally expressed via neuron-specific synapsin-1 promoter (schematics on left), localizes in neurons marked by Map-2 (and not in surrounding glia seen in DAPI panel), and co-localizes with the lysosomal protein Lamp2 in primary neuron cultures (immunofluorescence in middle panels). Expression of full length protein ${ }^{H A}$ Lamp1 ${ }^{\text {Myc }}$ is confirmed by immunoblotting for its epitope tags: N-terminal HA tag and C-terminal myc tag. (b) This lentiviral vector was used to generate Tg-HALamp1 ${ }^{\text {Myc }}$ mice via pronuclear injection of linearized DNA comprising the synapsin-1 promoter and the HALamp1 ${ }^{\text {Myc }}$ cDNA. (c) Tg-HALamp1Myc mice were PCR-genotyped using primer-pairs at the $\mathrm{N}$ - and the C-terminal ends of the ${ }^{\text {HALamp } 1 \text { Myc }}$ cDNA. Single insertion-site for the transgene was suggested by equal numbers of WT and Tg${ }^{H A}$ Lamp1 ${ }^{\text {Myc }}$ pups born per litter ( $n=11$ litters). There was also no effect of the transgene on birth-ratio and growth (indicated by weight gain; $n=5$ per group) of the Tg- ${ }^{H A}$ Lamp1 ${ }^{\text {Myc }}$ mice compared to WT mice. (d) Immunoblots show that HALamp1 ${ }^{\text {Myc }}$ protein is detectable in mouse brains by immunoblots against the $\mathrm{N}$-terminal HA tag and the C-terminal myc tag. All data represent means \pm SEM. n.s. = not significant, by 2-tailed Student's t-test for littermate ratio and by RM 2-way ANOVA for weight gain over age. Note: Further characterization of the Tg- HALamp ${ }^{\text {Myc }}$ mice is included in Supplementary Fig. S3. 
a

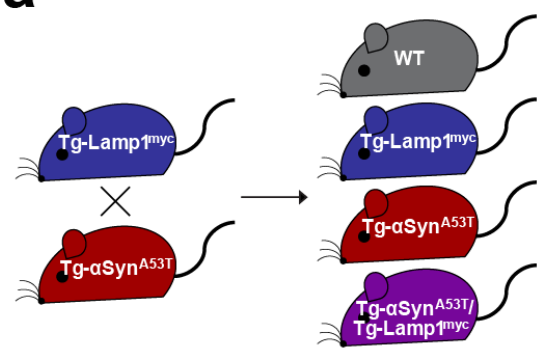

C

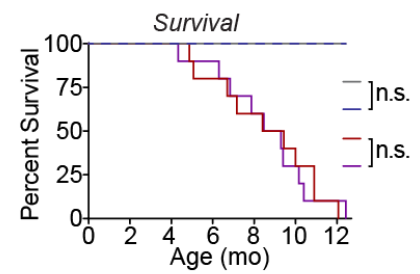

e

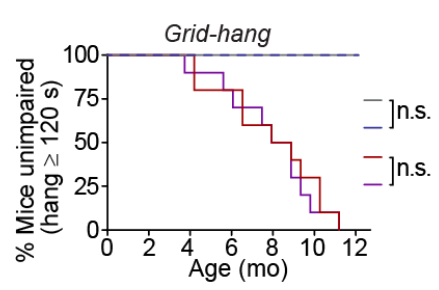

$\mathbf{f}$

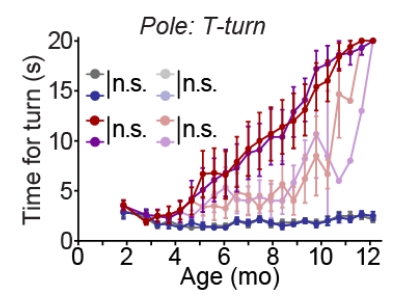

b

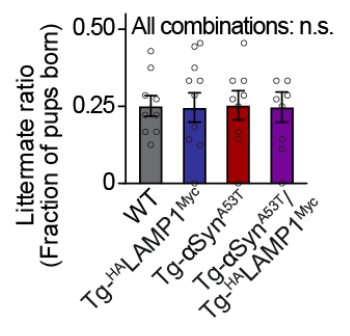

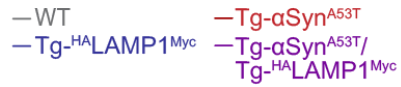

d
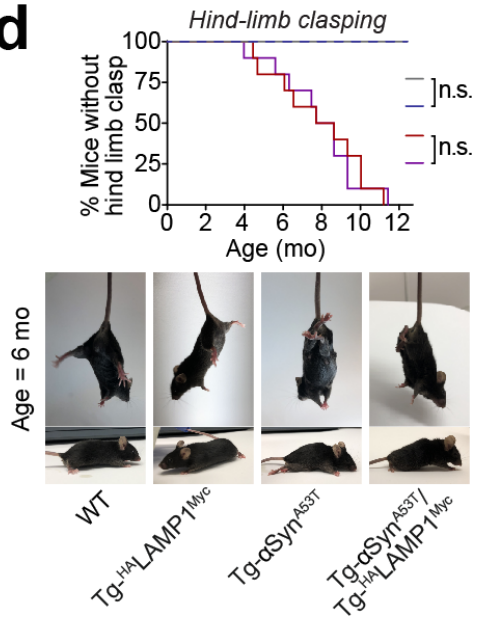

g

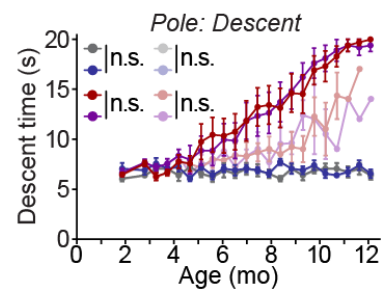

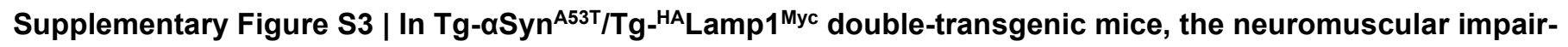
ments driven by $\alpha \mathrm{Syn}^{\mathrm{A53T}}$ transgene remain unaffected by the ${ }^{\mathrm{HA}}$ Lamp $1^{\text {Myc }}$ transgene.

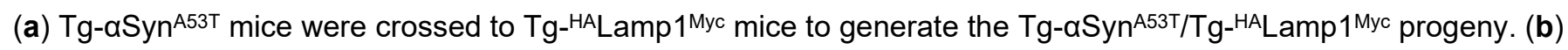

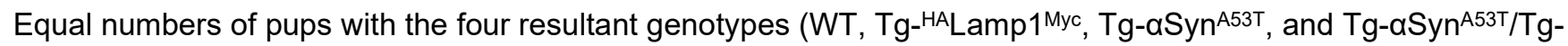

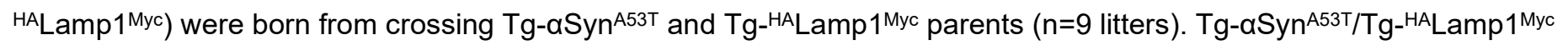
and $\mathrm{Tg}-\alpha \mathrm{Syn}^{\mathrm{A} 53 \mathrm{~T}}$ mice were indistinguishable in (c) lifespan, as well as in the onset and course of neuromuscular deterioration (d-g): Signs of progressive neuromuscular debility preceded death, as measured by (d) onset of hind-limb clasping, (e) grid-hang (limb strength), (f) T-turn on pole-test (bradykinesia), and (g) descent on pole test (motor coordination) ( $\mathrm{n}=10$ mice per group). (f-g) Mice that died were scored as $20 \mathrm{~s}$ (maximum measurement) for the rest of the trial - not as an actual measurement, but as a place holder. The same data are shown in the lighter shaded graphs without the $20 \mathrm{~s}$ placeholder, as dead animals are eliminated from the cohort over time. WT and Tg-HALamp1 ${ }^{\text {Myc }}$ mice showed no impairments in these measurements ( $n=5$ mice per group). ( $b$ and $\mathbf{f}-\mathbf{g}$ ) Data represent means \pm SEM. $n . s$. $=$ not significant, by RM 1-way ANOVA in (b), Log-rank (Mantel-Cox) test for comparisons of Kaplan-Meier curves (c-e), RM 2-way ANOVA (dark shaded graphs) and mixed-effects analysis (lighter shaded graphs) for pole turn and pole descent test (f-g). 
a

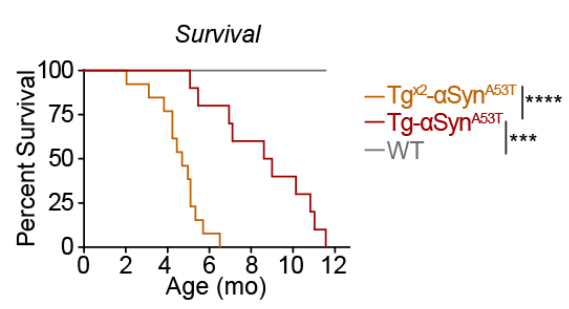

C

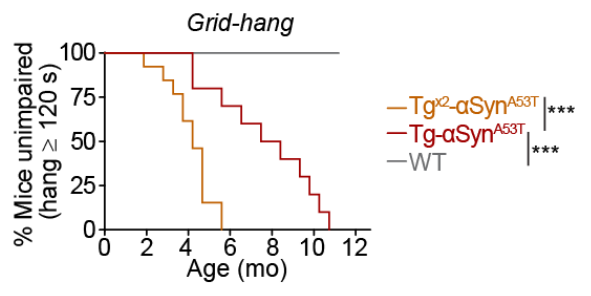

d

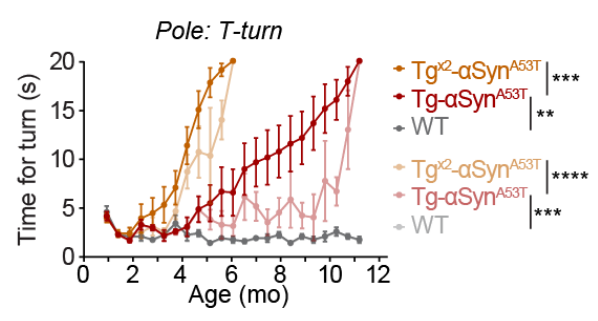

f

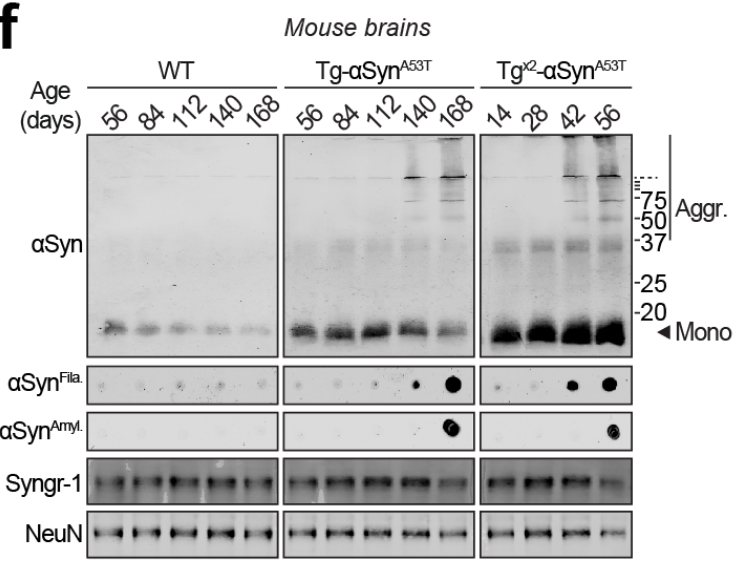

b
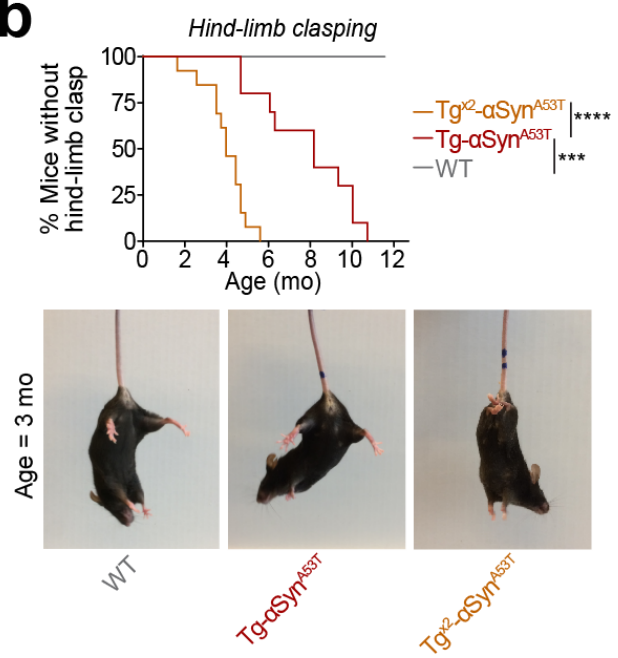

e

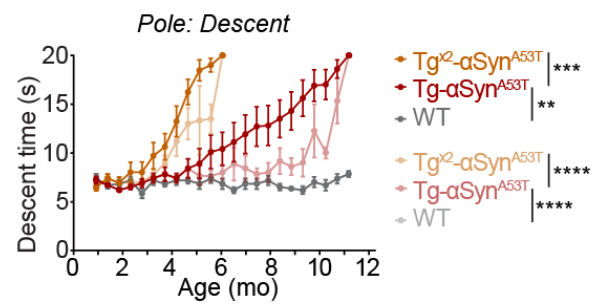

g

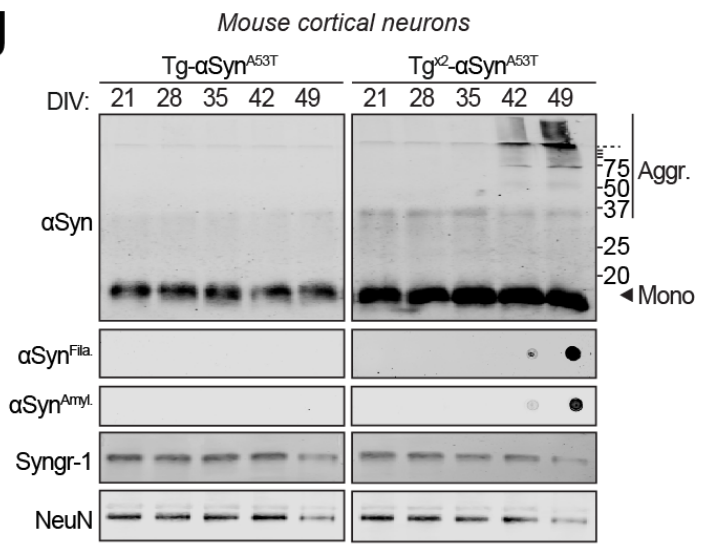

Supplementary Figure $\mathrm{S} 4$ | Characterization of the homozygous $\mathrm{Tg}^{\times 2}-\alpha \mathrm{Syn}^{\mathrm{A53T}}$ mice and primary neurons.

(a) Doubling the dose of $a S{ }^{A 53 T}$ in $\mathrm{Tg}^{\times 2}-\alpha \mathrm{Syn}^{\mathrm{A} 53 \mathrm{~T}}$ mice resulted in accelerated mortality, nearly halving the lifespan of $\mathrm{Tg}$ aSyn ${ }^{\mathrm{A53T}}$ mice. This is accompanied by accelerated onset of neuromuscular impairment in $\mathrm{Tg}^{\times 2}$ - $\alpha \mathrm{SSy}^{\mathrm{A} 53 \mathrm{~T}}$ mice, as measured by (b) onset of hind-limb clasping, (c) onset of grid-hang impairment (limb strength) (d) T-turn on pole-test (bradykinesia), and (e) descent on pole test (motor coordination). WT mice showed no impairments during the time course $(n=10$ $\mathrm{Tg}-\alpha \mathrm{Syn}^{\mathrm{A} 53 \mathrm{~T}} ; \mathrm{n}=13 \mathrm{Tg}^{\times 2}$ - $\alpha \mathrm{Syn}^{\mathrm{A} 53 \mathrm{~T}} ; \mathrm{n}=6 \mathrm{WT}$ mice). (d-e) Mice that died were scored as $20 \mathrm{~s}$ (maximum measurement) for the rest of the trial - not as an actual measurement, but as a place holder. The same data are shown in the lighter shaded graphs without the $20 \mathrm{~s}$ placeholder, as dead animals are eliminated from the cohort over time. (f) In addition to increased levels of aSyn monomer, $\alpha$ Syn aggregates are detected in $\mathrm{Tg}^{\times 2}-\alpha \mathrm{Syn}^{\mathrm{A}}{ }^{\mathrm{A} 3 \mathrm{~T}}$ brains much earlier than in $\mathrm{Tg}-\alpha \mathrm{Syn}^{\mathrm{A}}{ }^{53 \mathrm{~T}}$ brains.

(g) $\operatorname{Tg}^{\times 2}-\alpha S y n{ }^{A 53 T}$ primary neuron cultures recapitulate the accelerated accumulation of pathogenic aSyn species. (a-c) 
bioRxiv preprint doi: https://doi.org/10.1101/2021.04.10.439302; this version posted April 11, 2021. The copyright holder for this preprint (which was not certified by peer review) is the author/funder. All rights reserved. No reuse allowed without permission.

${ }^{* * *} P<0.001$ and ${ }^{* * * *} P<0.0001$ by Log-rank (Mantel-Cox) test. (d-e) Data represent means $\pm S E M,{ }^{* *} P<0.01 ;{ }^{* * *} P<0.001$; ${ }^{* * * *} \mathrm{P}<0.0001$ by RM 2-way ANOVA (dark shagged graphs) and mixed-effects analysis (lighter shaded graphs); analysis between $\operatorname{Tg}^{\times 2}-\alpha \mathrm{Syn}^{\mathrm{A}}{ }^{53 \mathrm{~T}}$ and $\mathrm{Tg}^{-\alpha S y n}{ }^{\mathrm{A} 53 \mathrm{~T}}$ is up to $6 \mathrm{mo}$. 
a
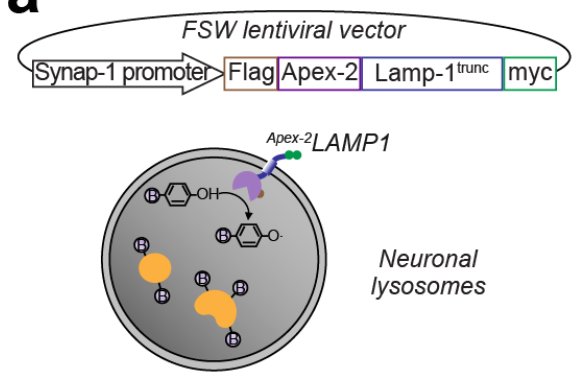

WT mouse cortical neurons (DIV14)

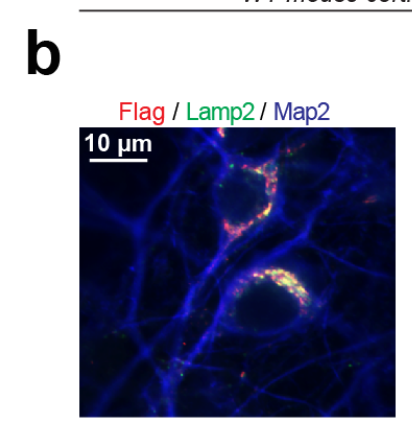

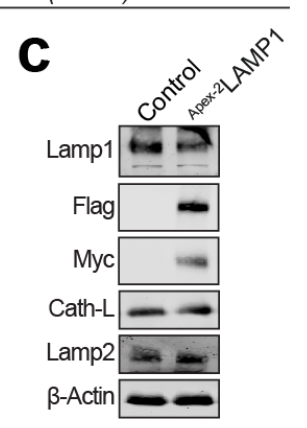

Supplementary Figure S5 | Generation of Apex-2Lamp1 chimeric protein for labeling lysosomal luminal proteins in primary neurons.

(a) Apex-2 was fused to the N-terminus of truncated Lamp1, and expressed via neuron-specific synapsin-1 promoter, allowing it to biotinylate lysosomal luminal proteins. (b) Lentivirally expressed Apex-2Lamp1 (infected on DIV 7, immunostained on DIV14) co-localizes with lysosomal protein Lamp2 in primary neurons. Neuronal somal and dendrites are marked by Map2. (c) Expression of Apex-2Lamp1 in primary neurons is confirmed by immunoblotting for its epitope tags: $\mathrm{N}$ terminal FLAG and C-terminal myc. 

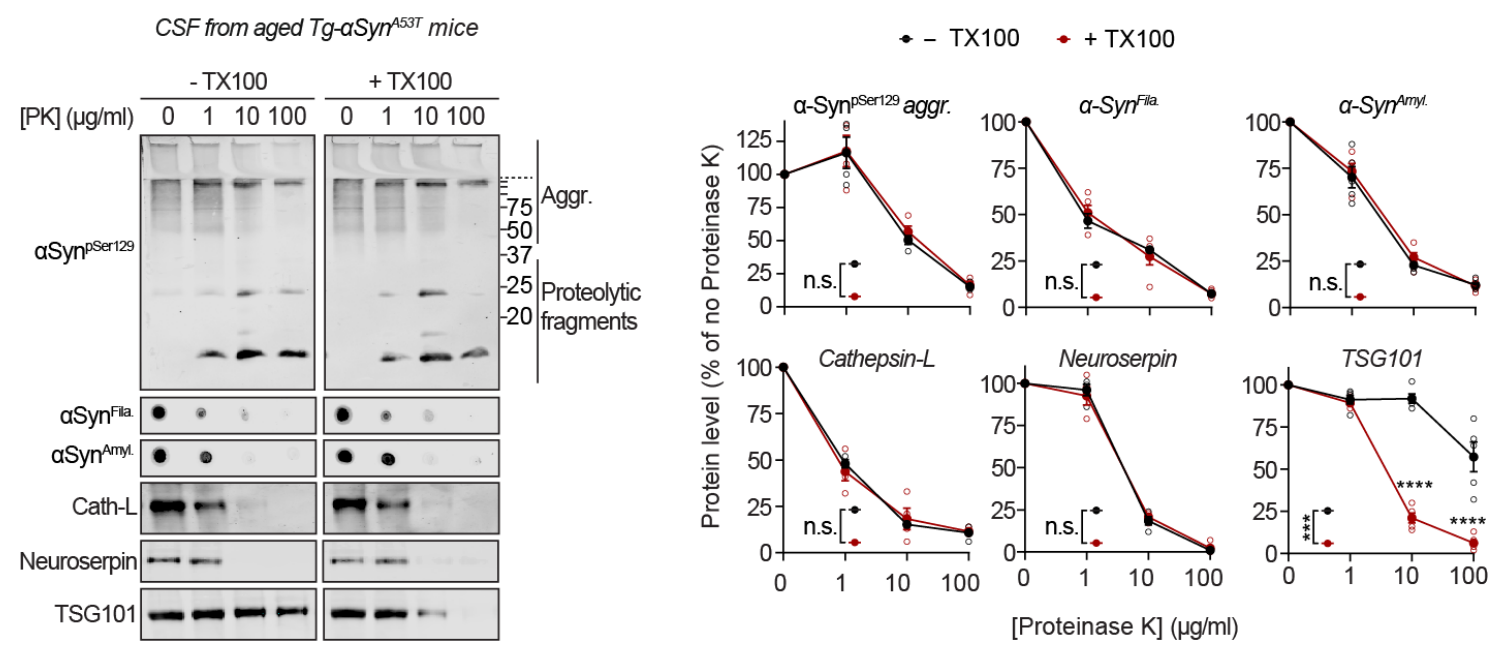

\section{Supplementary Figure S6 | Extracellular aSyn species in cerebrospinal fluid are not membrane-enveloped.}

Cerebrospinal fluid (CSF) collected from 6 month old Tg-aSyn ${ }^{A 53 T}$ mice were subjected to limited proteolysis using indicated concentrations of proteinase $\mathrm{K}(\mathrm{PK})$ in the absence or presence of $0.1 \%$ Triton $\mathrm{X}-100$. Immunoblots for aSyn protein levels ( $\alpha S{ }^{p S e r 129}, \alpha S y n^{A m y l}$, and $\alpha S y n^{\text {Fila }}$ ), cathepsin-L, TSG101, and neuroserpin $(n=4)$. All data represent means \pm SEM. Each ' $n$ ' is an independent proteolysis experiment. ${ }^{* * *} \mathrm{P}<0.001$; ${ }^{* * * *} \mathrm{P}<0.0001$ by RM 2-way ANOVA and Bonferroni post-test with multiple hypothesis correction. 
a

$T^{-H A}{ }^{\text {HA LAMP1MAc }}$ brain lysosomes (P25)

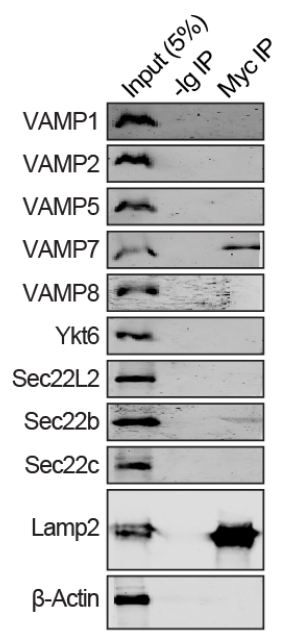

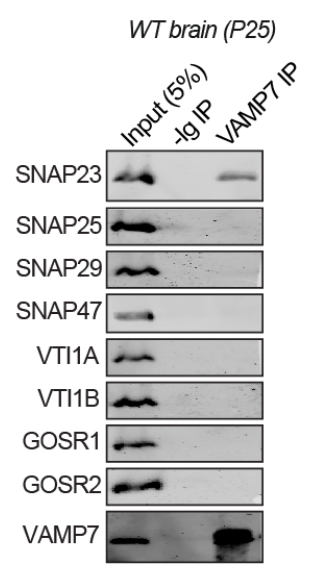
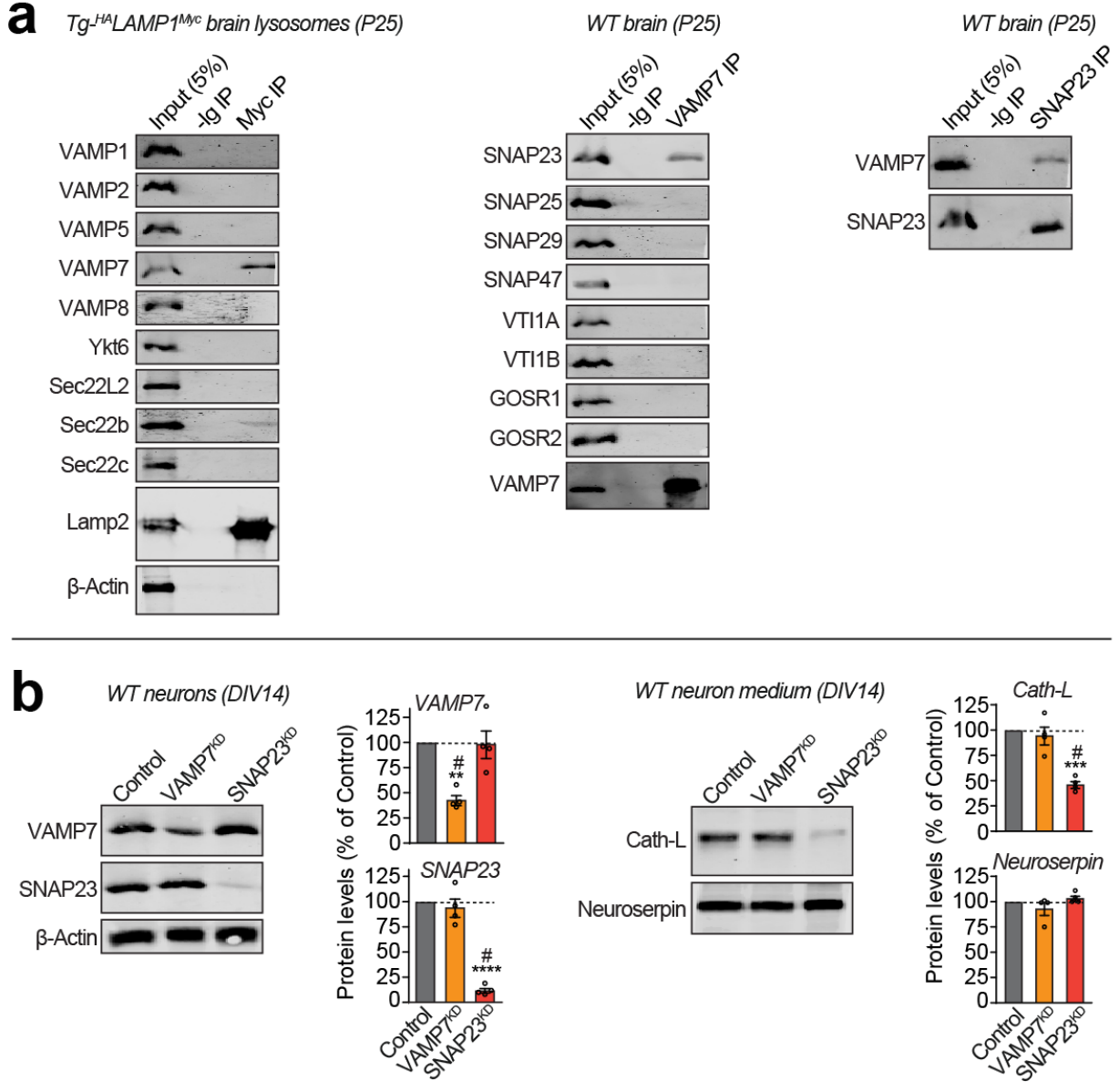

C
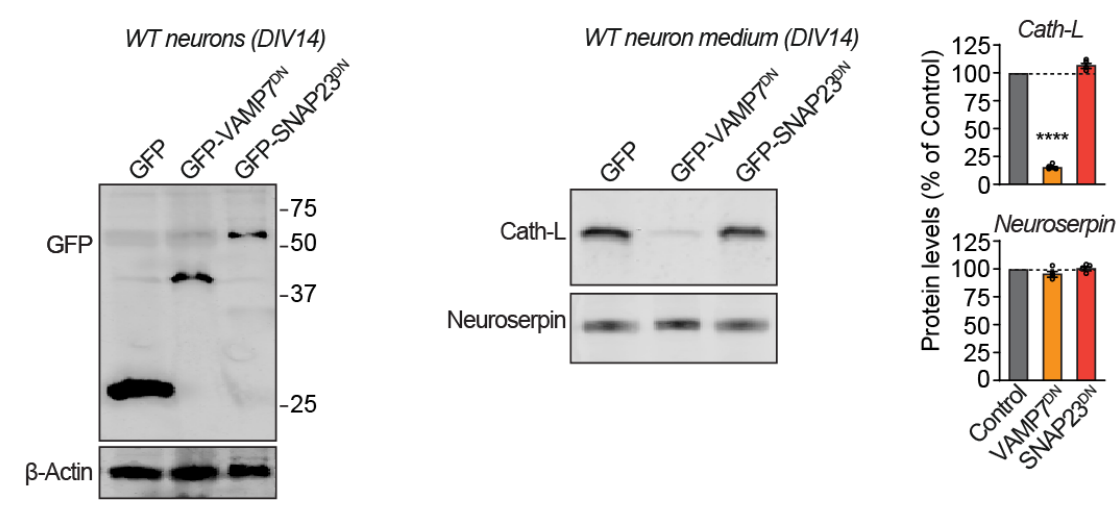

\section{Supplementary Figure S7 | SNARE-dependence of lysosomal exocytosis.}

(a) From Tg-HALamp1 ${ }^{\text {Myc }}$ mouse brains, we immunoisolated lysosomes (as in Fig. 2), followed by immunoblotting for the indicated vSNAREs (left panel). From wild type mouse brains, we immunoprecipitated (IP) VAMP7 and immunoblotted for the indicated co-IP'd Qb motif containing tSNAREs (middle panel). We then performed the inverse IP, where SNAP23 was IP'd and VAMP7 co-IP was tested by immunoblotting (right panel) (representative of $n=3$ ). (b) In WT neurons, shRNA knockdown constructs VAMP7 ${ }^{K D}$ and SNAP23 ${ }^{K D}$ were lentivirally expressed (Control = virus without shRNA), followed by quantitative immunoblotting of VAMP7 and SNAP23 levels $(n=4)$. Secreted levels of lysosome luminal protein cathepsin-L and the constitutively secreted protein neuroserpin were also quantified from the medium, and normalized to $\beta$-actin quantified from the respective culture lysate $(n=4)$. (c) In WT neurons, dominant-negative constructs GFP-VAMP7 ${ }^{K D}$ and GFP- 
SNAP23 ${ }^{\mathrm{KD}}$ were lentivirally expressed (Control $=$ GFP), followed by immunoblotting for GFP (representative of $n=4$ ). Quantification of cathepsin- $L$ and neuroserpin in the medium, normalized to $\beta$-actin in cell lysates $(n=4)$. All data represent means \pm SEM. Each ' $n$ ' is an independent immunoisolation/immunoprecipitation in (a) and an independently infected neuron culture in (b-c). In (b-c) ${ }^{* *} \mathrm{P}<0.01 ;{ }^{* * *} \mathrm{P}<0.001 ;{ }^{* * *} \mathrm{P}<0.0001$ by RM 1-way ANOVA with Dunnett multiple-comparison correction; and ${ }^{\#} \mathrm{P}<0.05$ by non-parametric Friedman test with Dunn's multiple-comparison adjustment. 


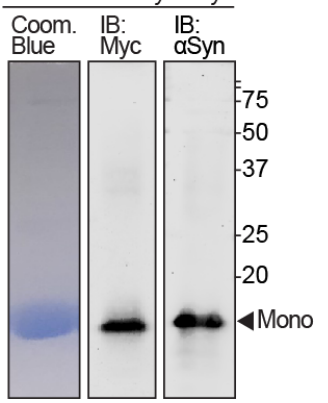

\section{Supplementary Figure S8 | Recombinant myc-aSyn.}

Purified recombinant myc-aSyn protein separated by SDS-PAGE, followed by Coomassie brilliant blue staining, as well as immunoblotting against myc and aSyn. 


\section{METHODS}

Mouse lines and husbandry

Mice were housed with a $12 \mathrm{~h} \mathrm{light/dark}$ cycle in a temperature-controlled room with free access to water and food. Animal husbandry and the experimental protocols used in this study were approved by the Institutional Animal Care and Use Committee (IACUC) at Weill Cornell Medicine.

CSPa knockout mice rescued by transgenic $\alpha S y n^{A 53 T}$ expression.

Transgenic mice that express $\alpha S_{5 y}{ }^{A 53 T}$ under the control of Thy-1 promoter (Chandra et al., 2005) were crossed to CSPa knockout mouse line (Fernández-Chacón et al., 2004) by breeding Tg- $\alpha \mathrm{Syn}^{\mathrm{A}}{ }^{53 \mathrm{~T}}$ to $\mathrm{CSPa}^{+/-}$mice. The early neurodegeneration and death of $\mathrm{CSPa}^{-/-}$mice was rescued by overexpressing aSyn ${ }^{\mathrm{A53T}}$ (Chandra et al., 2005). Rescued Tg-

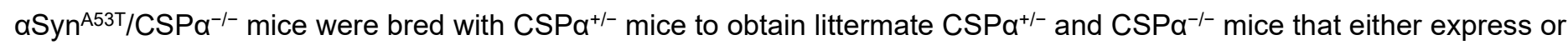
lack $\alpha S_{y n}{ }^{\mathrm{A} 53 \mathrm{~T}}$ transgene. $\mathrm{CSPa}^{+/-}$mice have no CSPa loss of function phenotype. Both, Tg-aSyn ${ }^{\mathrm{A} 53 \mathrm{~T}}$ and $\mathrm{CSPa}^{-/-} \mathrm{mouse}^{-2}$ lines are available from Jackson Laboratory: B6.Cg-Tg(THY1-SNCA*A53T)M53Sud/J (stock \# 008135) and B6.129S6Dnajc5tm1Sud/J (stock \# 006392), respectively.

Generation of Tg- ${ }^{H A}{ }_{\text {Lamp1 }}{ }^{\text {Myc }}$ mice.

The transgenic mice were derived from a lentiviral vector. The lentiviral vector included truncated synapsin-1 promoter followed by ORF including Lamp1 signal sequence - 2xHA epitope tag - rat Lamp1 - 6His - TEV cleavage site - 2xMyc epitope tag. This lentivirus vector was digested overnight at $37^{\circ} \mathrm{C}$ with $\mathrm{Pacl}$ and $\mathrm{Sphl}$ to create a $3.45 \mathrm{~kb}$ linear fragment for pronuclear microinjection. The cleavage product included truncated 5' LTR, the promoter and HALamp1Myc ORF (as described above), WPRE, and part of the bGH poly(A) signal. The enzymes were heat inactivated at $65^{\circ} \mathrm{C}$ for 20 minutes and the $3.45 \mathrm{~kb}$ band was purified/extracted using the Qiaquick gel extraction kit. The pronuclear injection was performed at Cornell University Stem Cell \&Transgenic Core Facility into B6(Cg)-Tyrc-2J/JxFVB embryos. 115 embryos were injected, and 76 embryos proceeded to the 2-cell stage. Of the 18 clones born, 2 positive clones (male founders 1 and 7 ) contained PCR-detectable full length ${ }^{H A}$ Lamp ${ }^{\mathrm{Myc}}$, followed by confirmation by immunoblotting for protein expression. The founder transgenic clones were bred to wild type female C57BL/6 mice. The genotyping primers amplify sequences either near the N-terminal end (F: 5'-CGCGACCATCTGCGCTG-3' and R: 5'-GCTGTGCCGTTGTTGTC-3'; product = 297 bp), or near the C-terminal end (F: 5'-GCACATCTTTGTCAGCAAGGCG-3' and R: 5'-GCAATAGCATGATACAAAGGC-3: product $=474 \mathrm{bp}$ ) of the insert.

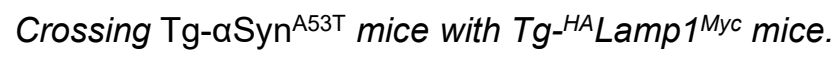

Neuron-specific ${ }^{\mathrm{HA} L a m p 1}{ }^{\mathrm{Myc}}$ transgenic mice were crossed to Tg- $\alpha \mathrm{Syn}{ }^{\mathrm{A} 53 \mathrm{~T}}$ mice, and bred either as single transgenics

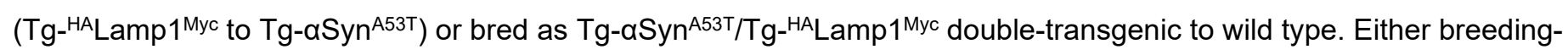

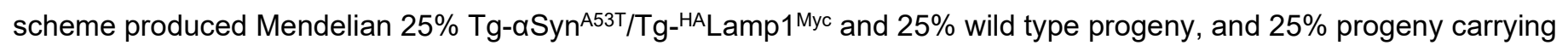
each transgene alone; indicating single copy or insertion site for each transgene, carried on distinct chromosomes. 
Enrichment of dextran-loaded lysosomes (dextranosomes), enhanced by mitochondrial swelling, was based on previously published techniques (Arai et al., 1991; Graham, 2001). Mice were anesthetized with isoflurane and intracranially injected with $10 \mu \mathrm{l}$ dextran-70 $(250 \mathrm{mg} / \mathrm{ml})$ into each cortex. $36 \mathrm{~h}$ later, mice were terminally anesthetized and perfused with homogenization buffer (HM = $10 \mathrm{mM}$ HEPES buffer $\mathrm{pH} 7.0$ with $0.25 \mathrm{M}$ sucrose and $1 \mathrm{mM}$ EDTA). The brain was dissected out, washed with $\mathrm{HM}$ and homogenized in $1.5 \mathrm{ml} \mathrm{HM}$. Homogenate was centrifuged at $340 \mathrm{gav}$ for 5 min, any floating fat layer was aspirated, pellet discarded, and the supernatant was re-centrifuged at $340 \mathrm{gav}_{\mathrm{av}}$ for $5 \mathrm{~min}$. The resultant post-nuclear supernatant was incubated with $1 \mathrm{mM} \mathrm{CaCl}_{2}$ for $5 \mathrm{~min}$ at $37^{\circ} \mathrm{C}$ to swell the mitochondria, in order to reduce mitochondrial contamination in the dextranosomal fractions. This solution was centrifuged at $10,000 \mathrm{gav}$ for 30 min to precipitate heavy organelles. The pellet containing swollen mitochondria, peroxisomes and dextranosomes was resuspended in $1 \mathrm{ml} \mathrm{HM}$ and layered over $9 \mathrm{ml}$ of $27 \% \mathrm{v} / \mathrm{v}$ Percoll in $0.25 \mathrm{M}$ sucrose, followed by centrifugation at 35,000 gav for $90 \mathrm{~min}$, to generate the Percoll gradient. $1 \mathrm{ml}$ fractions were collected from top, with dextranosomes expected near the bottom of the gradient. Fractions were centrifuged at $100,000 \mathrm{~g}_{\mathrm{av}}$ at for $1 \mathrm{~h}$ to pellet the Percoll particles. The supernatant above the Percoll pellet was concentrated using Amicon (10 kDa cutoff), and assayed.

\section{Assays for cathepsin- $D$, citrate synthase, and catalase activity}

Activities of enzymes contained within the lysosomes (cathepsin-D), mitochondria (citrate synthase), or peroxisomes (catalase) were measured from the Percoll gradient fractions dissolved in 0.1\% Triton X-100 (final). Cathepsin-D activity was assayed using ab65302 kit (Abcam) according to the manufacturer's protocol. Each fraction was incubated with the cathepsin-D substrate GKPILFFRLK(Dnp)-D-R-NH labeled with MCA at $37^{\circ} \mathrm{C}$ for $1 \mathrm{~h}$ in the dark. Fluorescence was measured at Ex: $328 \mathrm{~nm}$, Em: $460 \mathrm{~nm}$, in a solid white 96-well plate (Costar). Citrate synthase activity was assayed using ab239712 kit (Abcam) according to the manufacturer's protocol, and absorbance at $412 \mathrm{~nm}$ was measured in clear bottom 96-well plate (Costar). Catalase activity was assayed using ab83464 kit (Abcam) according to the manufacturer's protocol, and absorbance at $570 \mathrm{~nm}$ was measured in clear bottom 96-well plate (Costar). Synergy H1 Hybrid Reader was used for all three enzyme assays.

Neuromuscular behavior tests and survival study

Pole test. The pole test was performed based on the method established by Ogawa et al. (Ogawa et al., 1985). Animals were positioned with their head upward near the top of a wooden dowel $(1 \mathrm{~cm}$ in diameter and $50 \mathrm{~cm}$ high). The time taken until they turned completely downward (defined as a "T-turn", indicative of bradykinesia) and time taken after the Tturn to descend to the bottom (indicative of motor coordination) were recorded. All animals were trained three times before performing the test. The maximum time allowed for each measurement was $20 \mathrm{~s}$. If the animal could not turn, or fell during descent, the experiment was repeated; and if the animal again could not turn or fell during descent, the time for the activity was recorded as the maximum $20 \mathrm{~s}$. Grid hang test. As in (Burré et al., 2010), animals were placed on top of a wire mesh grid $(1.27 \mathrm{~cm} \times 1.27 \mathrm{~cm})$. The grid was then shaken lightly to cause the mouse to grip the wires with all four limbs, and then turned upside down. The mesh was held approximately $20 \mathrm{~cm}$ above the home cage litter, high enough to prevent the mouse of easily climbing down but not to cause harm in the event of a fall. A stopwatch was used to record the time taken by the animal to fall off the grid. Three trials per mouse were performed with a 1 min inter-trial interval. The highest time of the 3 trials was assigned as the time for each animal to fall. After a maximum hang time of $120 \mathrm{~s}$, mice 
were removed from the grid. Hind limb clasping. Mice were lifted by tail for $20 \mathrm{~s}$. If an animal retracted its hind limbs toward the abdomen and held them there, it was scored positive for hind limb clasping. Survival study. End point for survival study was reaching neuromuscular debility (e.g. paresis) which could make it difficult for the animal to get to food or water. At that time, the animal was euthanized according to the approved protocols.

\section{Immuno-isolation of lysosomes from mouse brains}

Immunoprecipitation was used to isolate lysosomes from Tg-HALamp1 ${ }^{\text {Myc }}$ mouse brains. Mouse brains were lysed in PBS without detergent and supplemented with EDTA-free protease inhibitor cocktail (Thermo Fisher). The post-nuclear supernatant was incubated with anti-myc magnetic beads (with covalently cross-linked clone $9 \mathrm{E} 10 \mathrm{mAb}$; Pierce) for $2 \mathrm{~h}$ at $4^{\circ} \mathrm{C}$, followed by 3 washes in PBS. The precipitated material was eluted in non-reducing $2 x$ Laemmli sample buffer at room temperature, followed by immunoblotting.

\section{Mouse CSF collection and limited proteolysis}

6 month old Tg-aSyn ${ }^{\mathrm{A} 53 \mathrm{~T}}$ mice were terminally anesthetized with isoflurane, and CSF was collected by exposing cisterna magna (Lim et al., 2018), and using syringe aspiration (Hamilton $10 \mu \mathrm{l}$ syringe). Samples with visible signs of blood were discarded. Following CSF collection, the mice were secondarily euthanized by cervical dislocation and brain collected for unrelated experiments. Due to low and/or variable volume of CSF collected in different attempts, multiple littermate mice were tapped and the CSF was pooled prior to proteolysis experiments.

Fresh pooled CSF at room temperature was aliquoted into 2 equal portions, and equal volume of either PBS or PBS containing Triton X-100 (0.1\% final) was added to each tube and incubated for $10 \mathrm{~min}$. Samples were then mixed with equal volume of Proteinase $\mathrm{K}\left(0,1,10\right.$, or $100 \mu \mathrm{g} / \mathrm{ml}$ final) from $100 \mathrm{x}$ stocks in sterile water, and incubated for $1 \mathrm{~h}$ at $15^{\circ} \mathrm{C}$ (to minimize membrane permeability). Proteolysis was immediately stopped by adding $5 x$ Laemmli sample buffer with PMSF (1 $\mathrm{mM}$ final), followed by immunoblotting.

\section{Immunoprecipitation}

For co-immunoprecipitation of SNARE proteins, brain homogenate was lysed in PBS containing $0.1 \%$ Triton X-100 and supplemented with EDTA-free protease inhibitor cocktail (ThermoFisher). Postnuclear supernatant was incubated for $2 \mathrm{~h}$ at $4{ }^{\circ} \mathrm{C}$ with the antibody, and then for another $1 \mathrm{~h}$ following the addition of protein-G Sepharose (GE Healthcare). Protein bound to antibody beads was washed five times with lysis buffer at $4{ }^{\circ} \mathrm{C}$, and eluted in $2 x$ Laemmli sample buffer. Eluent was boiled for $20 \mathrm{~min}$ to disassemble SNARE complexes, followed by immunoblotting.

\section{Long term primary neuron culture from $\operatorname{Tg}^{2 \mathrm{x}}-\alpha \mathrm{Syn}^{\mathrm{A53T}}$ mice}

Primary cultures were based on modification of our neuron culture protocol (Naseri et al., 2020) with published procedures for long term cultures (Lesuisse and Martin, 2002). Neonatal (P0) cerebral cortices were isolated and dissected in cold HBSS. Cortices were dissociated by trypsinization (0.05\% trypsin-EDTA for $10 \mathrm{~min}$ at $37^{\circ} \mathrm{C}$ ), triturated with a siliconized pipette and plated onto poly-I-lysine-coated 24-well plastic dishes at high density $\left(800-1000 \mathrm{cells} / \mathrm{mm}^{2}\right.$; each experiment 
had the same cell density across plates), in DMEM (Invitrogen) supplemented with $5 \%$ horse serum (Invitrogen) and $1 \%$ penicillin-streptomycin (Life Technologies). Humidity was enhanced by adding autoclaved water in spaces between the wells, and the plates were not disturbed for at least 5 days. On day 5-7, medium was changed to serum-free neurobasal medium (Life Technologies) supplemented with 2\% B27 (Life Technologies) or N21-MAX (R\&D Systems), and neurons were infected at the same time with lentiviruses. $50 \%$ of the medium was changed every 7 days. Neuronal cultures were maintained for up to 7 weeks (DIV 49).

$\mathrm{Tg}^{2 \mathrm{x}}$ - $\alpha \mathrm{Syn}^{\mathrm{A53T}}$ mouse breeders were derived from $\mathrm{Tg}-\alpha \mathrm{Syn}^{\mathrm{A53T}}$ mice crossed to each other. However, this line was difficult to breed due to short survival and unreliable breeding output. Alternatively, we cultured cortices from all of the pups from

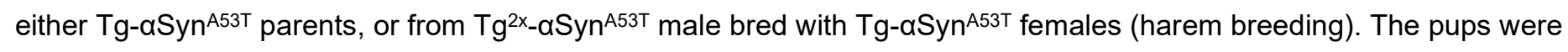
tested for homozygosity by cerebellar immunoblots against $\alpha$ Syn. Only $\operatorname{Tg}^{2 \mathrm{x}}-\alpha \mathrm{Syn}^{\mathrm{A} 53 \mathrm{~T}}$ cultures were maintained through to the DIV 5 steps of medium replacement and lentiviral infection.

Media from 49 day old neurons was spun through 5 um mini strainer (pluriselect) to exclude any debris, followed by concentration on $10 \mathrm{kDa}$ Amicon (Millipore).

\section{Lentivirus production and transduction}

HEK293T cells were co-transfected with the lentiviral vector plasmid, with the HIV-1 lentiviral packaging constructs pRSVREV and pMDLg/pRRE (Dull et al., 1998), and the vesicular stomatitis virus-G expression plasmid pHCMVG (Yee et al., 1994). The virus-containing culture supernatant was collected $48 \mathrm{~h}$ post-transfection and was concentrated by centrifugation at 50,000 gav for $90 \mathrm{~min}$. The viral pellet was resuspended in neuronal medium (at 1/10 of the pre-centrifugation volume). All lentiviruses used in a single experiment were prepared together. Neurons were infected on DIV 5-7, and harvested for experiments at times described in figure captions.

\section{Immunofluorescence in neurons}

For immunofluorescent labeling of cortical neuron cultures, cells on coverslips were washed with PBS + $1 \mathrm{mM} \mathrm{MgCl} 2$ and fixed in $4 \%$ paraformaldehyde for $30 \mathrm{~min}$ at room temperature. Cells were permeabilized for $5 \mathrm{~min}$ in PBS $+0.1 \%$ Triton X-100, and blocked for $20 \mathrm{~min}$ in PBS $+5 \%$ BSA (blocking buffer). Coverslips were then incubated in primary antibodies in blocking buffer overnight at $4{ }^{\circ} \mathrm{C}$. Following five washes, coverslips were incubated with secondary antibodies labeled with Alexa 488 and/or Alexa 546 (Life Technologies) in blocking buffer containing DAPI, for 1-2 h, followed by five PBS washes and mounting on slides in Fluormount G (ThermoFisher). Images were acquired on a Nikon H550L microscope.

\section{SNARE shRNA knockdown and dominant-negative strategies}

Once VAMP7 and SNAP23 were identified as cognate lysosomal SNAREs, in agreement with prior studies (Rao et al., 2004), we modulated their function via shRNA knockdown and dominant-negative strategies: Based on pre-characterized target sequences and hairpins described on the Broad Institute Genetic Perturbation Platform, mouse VAMP7 and 
SNAP23 shRNAs were cloned into L309 lentiviral vector to be expressed under human H1 promoter. For VAMP7, the target sequence was 5'-TTACGGTTCAAGAGCACAAAC-3', and for SNAP23, the target sequence was 5'-CAACCGAGCCGGATTACAAAT-3'. Rescue expression of human SNAP23 from FUW vector (Lois et al., 2002) was not affected by the anti-mouse shRNA target sequence.

To express a dominant negative VAMP7 fragment, rat VAMP7/TI-VAMP truncated at the C-terminal end (Ala ${ }^{2}$-Asn ${ }^{120}$ fragment expressed) (Martinez-Arca et al., 2000) was expressed as an eGFP chimera (GFP-VAMP72-120) via FUGW lentiviral vector (Lois et al., 2002).

To generate a dominant negative version of SNAP23, the last eight residues of human SNAP23 were deleted (Asp ${ }^{2}-\mathrm{Ala}^{203}$ segment expressed) mimicking the dominant negative SNAP25 ${ }^{1-197}$ fragment generated by Botulinum toxin-A cleavage (Huang et al., 1998) and expressed as an eGFP chimera (GFP-SNAP232-203) from FUGW lentiviral vector (Lois et al., 2002).

\section{Proximity-labeling of lysosomal contents}

To target Apex-2 to the lysosomal lumen, Apex-2 was fused at the N-terminus to the transmembrane domain of rat Lamp1. The Apex-2Lamp1 construct consists of: Lamp1 signal sequence - Flag tag - APEX2 - rat Lamp1 truncation including transmembrane domain plus C-terminal cytosolic tail with lysosome-targeting motif (Lamp1370-407 including the targeting motif ${ }^{403} \mathrm{GYQTI}{ }^{407}$ ) - $6 \mathrm{His}$ - TEV - Myc tag, generating a $36 \mathrm{kDa}$ protein.

Apex-2Lamp1 was lentivirally expressed via a truncated synapsin-1 promoter in $\mathrm{Tg}^{\times 2}-\alpha \mathrm{Syn}^{\mathrm{A} 53 \mathrm{~T}}$ primary neurons by infection on DIV7. On DIV 49 , cells were incubated at $37^{\circ} \mathrm{C}$ for $1 \mathrm{~h}$ in medium containing $500 \mu \mathrm{M}$ biotin tyramide, plus a lysosomal $\mathrm{pH}$-raising cocktail (10 nM NH $4 \mathrm{Cl}$ and $100 \mathrm{nM}$ Bafilomycin A1) to enhance the reaction. $\mathrm{H}_{2} \mathrm{O}_{2}$ was added at a final concentration of $1 \mathrm{mM}$ for exactly $1 \mathrm{~min}$ at room temperature. Reaction was quenched for $30 \mathrm{sec}$ with PBS $+1 \mathrm{mM} \mathrm{MgCl} 2 \mathrm{contain}-$ ing antioxidants (5 mM Trolox, $10 \mathrm{mM}$ ascorbic acid). Cells were washed with PBS $+1 \mathrm{mM} \mathrm{MgCl}_{2}$, and lysed in PBS + $0.1 \%$ Triton-X 100, and biotinylated proteins were precipitated on streptavidin-magnetic beads (Dynabeads; Thermo), with 3 washes before elution in $2 x$ Laemmli sample buffer.

To chase biotin-labeled proteins, $\mathrm{Tg}^{\times 2}$ - $\alpha \mathrm{S} y \mathrm{n}^{\mathrm{A} 53 \mathrm{~T}}$ primary neurons lentivirally expressing Apex-2 Lamp1, were subjected to biotin labeling on DIV 47, similar to above, except using $0.5 \mathrm{mM} \mathrm{H}_{2} \mathrm{O}_{2}$ to reduce toxicity. The reaction was quenched with $37^{\circ} \mathrm{C}$ medium containing antioxidants ( $5 \mathrm{mM}$ Trolox, $10 \mathrm{mM}$ sodium ascorbate, and $5 \mathrm{mM}$ glutathione). 10 minutes later, the medium was replaced, containing $2.5 \mathrm{mM}$ glutathione. $48 \mathrm{~h}$ later, the culture-medium was collected, and biotinylated proteins were precipitated on streptavidin-magnetic beads (Dynabeads; Thermo), with 3 washes before elution in $2 x$ Laemmli sample buffer.

\section{Purification and in vitro aggregation of recombinant myc- $\alpha$ Syn}

Recombinant myc-aSyn was expressed and purified essentially as in (Burré et al., 2015). Full-length human aSyn cDNA containing an N-terminal myc epitope-tag was inserted into a modified pGEX-KG vector (GE Healthcare), after a TEV protease recognition site to allow cleavage from GST (myc-aSyn contains an extra N-terminal glycine after cleavage with 
TEV protease). BL21 strain of $E$. coli bacteria transformed with this plasmid were grown to optical density 0.6 (at $600 \mathrm{~nm})$, and protein expression was induced with $0.05 \mathrm{mM}$ isopropyl $\beta$-D-thiogalactoside (IPTG) for 6 hours at room temperature. Bacteria were pelleted by centrifugation for $20 \mathrm{~min}$ at $4000 \mathrm{rpm}$, and then resuspended in solubilization/lysis buffer, PBS containing $0.5 \mathrm{mg} / \mathrm{ml}$ lysozyme, $1 \mathrm{mM}$ PMSF, DNase, and an EDTA-free protease inhibitor mixture (Roche). Bacteria were further broken by sonication, and insoluble material was removed by centrifugation for $30 \mathrm{~min}$ at $7000 \mathrm{gav}$ at $4^{\circ} \mathrm{C}$. Protein was affinity-bound to glutathione Sepharose beads (GE Healthcare) by incubation overnight at $4^{\circ} \mathrm{C}$, followed by TEV protease (Invitrogen) cleavage for 6 hours at room temperature. The His-tagged TEV protease was removed by incubation with Ni-NTA (Qiagen) overnight at $4^{\circ} \mathrm{C}$. Protein concentration was assessed using the bicinchoninic acid (BCA) assay (Thermo Fisher Scientific).

For the aggregation studies, $10 \mu$ of concentrated extracellular medium from neuronal cultures - collected over 7 days (DIV 42-49), spun through $5 \mu \mathrm{m}$ strainer (Puriselect), and concentrated 10x using $10 \mathrm{kDa}$ cutoff Amicon (Millipore) - was added to $40 \mu \mathrm{l}$ of recombinant myc- $\alpha$ Syn (to $4 \mu \mathrm{g} / \mu \mathrm{l}$ final concentration) in PBS with protease inhibitors. This mixture was incubated at $37^{\circ} \mathrm{C}$ with shaking at $300 \mathrm{rpm}$, while sample aliquot were taken at indicated time periods and frozen at $-80^{\circ} \mathrm{C}$. At the end of final incubation time-point, all the samples were thawed and measured together.

$5 \mu \mathrm{l}$ of sample was mixed with $95 \mu \mathrm{l}$ of $100 \mu \mathrm{M} \mathrm{K114}$ (Santa Cruz Biotechnology) in $100 \mathrm{mM}$ glycine-NaOH, pH 8.45, and K114 fluorescence was measured at Ex: $390 \mathrm{~nm}$, Em: $535 \mathrm{~nm}$, in a solid white 96-well plate (Costar) using a plate reader (Synergy H1 Hybrid Reader, BioTek). Thioflavin-T measurement was done similarly, by mixing $5 \mu$ sample with $95 \mu$ l of $25 \mu \mathrm{M}$ Thioflavin-T (Sigma) in PBS, pH 7.4, and fluorescence was measured at Ex: $450 \mathrm{~nm}$, Em: $485 \mathrm{~nm}$. After fluorometry, the same samples were dissolved in Laemmli sample buffer and used for SDS-PAGE or dot blots, followed by immunoblotting.

\section{SDS-PAGE and quantitative immunoblotting}

For SDS-PAGE, $10-15 \%$ Laemmli gels $(10.3 \% \mathrm{~T}$ and $3.3 \% \mathrm{C})$ were used to separate proteins on Bio-Rad apparati. $1 \mathrm{mM}$ final DTT was used in SDS Laemmli buffer. Proteins were transferred onto nitrocellulose (pore-size $=0.45 \mu \mathrm{m}$; GE Healthcare) and blocked with $5 \% \mathrm{w} / \mathrm{v}$ fat-free dry milk in tris buffered saline, $\mathrm{pH} 7.5$ supplemented with $0.1 \%$ Tween 20 (TBS-T). Immunoblotting was performed by incubating the blocked membranes with primary antibodies in blocking buffer for 8-16 hours. Following 5 washes with TBS-Tween 20 (0.1\%). Blots were incubated with secondary antibodies (goat antirabbit conjugated to IRDye $600 \mathrm{RD}$ or $800 \mathrm{CW}$; LI-COR) at 1/5000 in blocking buffer for 1-3 hours. Immunoblots were washed $5 x$ with TBS-T and dried, then scanned on Odyssey CLx (LI-COR) and quantified using Image-Studio software (LI-COR).

For ATP5G immunoblots, following the transfer, nitrocellulose membranes were dried and fixed for 15 min at room temperature in 1\% paraformaldehyde in PBS (Ikegaki and Kennett, 1989). Membranes were then washed 3x with TBS-T and treated as above.

For dot blots, samples were dotted onto dry nitrocellulose membrane (if sample volume $<5 \mu \mathrm{l}$ ) or on a PBS wetted nitrocellulose membrane under vacuum (if volume was $>5 \mu \mathrm{l}$ ), and allowed to dry. The membrane was then immunoblotted as above. 
Antibody list

ß-Actin: Sigma (A1978); A11 oligomers: Stressmarq (SPC-506D); ATP5G: Abcam (ab181243); Calreticulin: Thermo Fisher (OTI15F5) and Novus (NB600-103); Cathepsin-L: Novus (JM10-78); CD81: Novus (SN206-01); EEA1: Thermo Fisher (MA5-14794); Flag: Sigma Cl. M2 (F3165); GAPDH: Cell Signaling Cl. 14C10 (2118); GFP: Takara Cl. JL8 (632381) and Invitrogen (A11122); GluN2B/NR2B: Cell Signaling CI. D15B3 (4212); GOSR1: Abcam (ab53288); GOSR2: ProteinTech (12095-1-AP); HA epitope: Abcam Cl. 16B12 HA.11 (ab130275); Histone H3: Cell Signaling Cl. 96C10 (3638); Hsp60: Abcam (ab5479); Lamp-1: DSHB CI. H4A3 and Cl. 1D4B DSHB Rat mono; Lamp-2: Cl. ABL-93 DSHB Rat mono; Map-2: Millipore (AB5622); Myc epitope: DSHB Cl. 9E10 and Sigma (C3956); Na/K-ATPase: DSHB Cl. A6F; NeuN: Millipore Cl. A60 (Mab377); Neuroserpin: Abcam (ab33077); Pex3: Thermo Fisher (PA5-37012); Pex13: Sigma (ABC143); Sec22b: SYSY (186 003); Sec22c: Novus (NBP1-30760); Sec22L2: MyBiosource.com (MBS8507611); SNAP23: Santa Cruz (sc166244) and Sudhof lab P914; SNAP25: Biolegend Cl. SMI81 (836304) and Sudhof lab P913; SNAP29: SYSY (111303); SNAP47: SYSY (111403); synaptogyrin-1: SYSY (103 002); aSyn: BD Transduction (610787) and Sudhof Lab T2270; aSyn

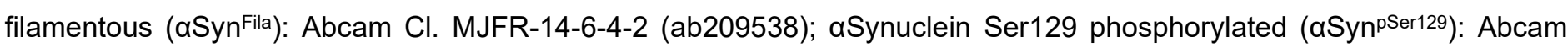
(ab51253); TGN38: BD Transduction (610899); TIM23: BD Transduction (611223); TSG101: Abcam (ab125011); $\alpha-T u b u l i n:$ DSHB CI. 12G10; VAMP1: SYSY (104 002); VAMP2: SYSY (104 202); VAMP5: SYSY (176 003); VAMP7/TiVAMP: SYSY (232 011) and SYSY (232 003); VAMP8: SYSY (104 302); VTI1A: SYSY (165 002); VTI1B: SYSY (164 002); Ykt6: Abcam (ab241382).

\section{Statistical analyses}

Each " $n$ " consisted of reagents produced in parallel (e.g., purified proteins, lentivirus production, culturing neurons, brain or CSF harvested from mice euthanized together) and experiments performed in parallel (e.g. lentiviral infection, sample collection for immunoprecipitation and immunoblotting, mouse behavior recorded on same day etc.). Experiments were quantified using parametric as well as non-parametric statistical tests when warranted (see the tests described below and in figure captions). Randomization and coding of mice or samples was done by different investigator(s) than the investigator who did quantification and analysis. All analyses are performed using GraphPad Prism 8 and described in the figure legends. Column analyses of data with more than 2 groups depicted in bar graphs (e.g. aSyn species in lysosomal fractions and aSyn species in extracellular media) were analyzed by repeated measures (RM) 1-way ANOVA with Dunnett multiplecomparison correction, as well as using the non-parametric Friedman test with Dunn's multiple-comparison adjustment. For comparison of only two groups (e.g. such as for littermate ratio of WT and Tg- HA Lamp1Myc cross) 2-tailed Student's t-test was used. For time-course experiments (e.g. accumulation of aSyn over age and seeding of recombinant aSyn) the curves were analyzed using RM 2-way ANOVA in comparing the overall curve, plus the Bonferroni post-test with multiple hypothesis correction to compare data at each time-point independently or to examine more than two groups. Graphs depicting the correlation (e.g. between the levels of pathogenic aSyn species released versus the levels of cathepsin- $L$ released in the medium) were analyzed via Pearson's correlation and shown with linear regression lines, as well as the $95 \%$ confidence interval using dotted lines. Kaplan-Meier curves (e.g. survival, onset of hind-limb clasping, and onset of grid-hang impairments) were compared via Log-rank (Mantel-Cox) test. For vertical pole studies, due to decreasing sample size with each mouse death, two separate curves were graphed to represent each of the pole-turn and the pole-descent data. Mice that died were scored as $20 \mathrm{~s}$ (maximum measurement) for rest of the trial - not as an actual measurement, but as a place 
bioRxiv preprint doi: https://doi.org/10.1101/2021.04.10.439302; this version posted April 11, 2021. The copyright holder for this preprint (which was not certified by peer review) is the author/funder. All rights reserved. No reuse allowed without permission.

holder. Data with 20 s placeholder were analyzed by RM 2-way ANOVA and data without the 20 s placeholder were analyzed via mixed effects analysis. 


\section{ACKNOWLEDGEMENTS}

We thank Dr. Tom Südhof for kindly sharing the CSPa knockout and Tg- $\alpha \mathrm{Syn}^{\mathrm{A} 53 \mathrm{~T}}$ mouse lines, as well as antibodies against $\alpha$ Syn, SNAP-23, and SNAP-25.

\section{FUNDING}

This work was supported by NIH F31 studentship (NS098623, to N.N.N.), grants from Alzheimer's Association (NIRG-15363678 to M.S.), American Federation for Aging Research (New Investigator in Alzheimer's Research Grant, to M.S.), NIH National Institute for Neurological Disorders and Stroke (1R01NS102181 and 1R01NS113960, to J.B.; and 1R01NS095988, to M.S.), NIH National Institute for Aging (1R01AG052505, to M.S.).

\section{AUTHOR CONTRIBUTIONS}

Y.X.X., N.N.N., J.F., P.K., Y.N., J.B., and M.S. designed, performed and analyzed all experiments. Y.X.X., N.N.N., J.B., and M.S. wrote the manuscript. M.S. conceived the project and directed the research.

\section{COMPETING INTERESTS}

The authors declare no competing interests. 


\section{REFERENCES}

Abounit, S., Bousset, L., Loria, F., Zhu, S., de Chaumont, F., Pieri, L., Olivo-Marin, J.C., Melki, R., and Zurzolo, C. (2016). Tunneling nanotubes spread fibrillar $\alpha$-synuclein by intercellular trafficking of lysosomes. The EMBO journal 35, $2120-2138$.

Ahn, K.J., Paik, S.R., Chung, K.C., and Kim, J. (2006). Amino acid sequence motifs and mechanistic features of the membrane translocation of alpha-synuclein. J Neurochem 97, 265-279.

Arai, K., Kanaseki, T., and Ohkuma, S. (1991). Isolation of highly purified lysosomes from rat liver: identification of electron carrier components on lysosomal membranes. J Biochem 110, 541-547.

Baba, M., Nakajo, S., Tu, P.H., Tomita, T., Nakaya, K., Lee, V.M., Trojanowski, J.Q., and Iwatsubo, T. (1998). Aggregation of alpha-synuclein in Lewy bodies of sporadic Parkinson's disease and dementia with Lewy bodies. The American journal of pathology 152, 879-884.

Benitez, B.A., Alvarado, D., Cai, Y., Mayo, K., Chakraverty, S., Norton, J., Morris, J.C., Sands, M.S., Goate, A., and Cruchaga, C. (2011). Exome-sequencing confirms DNAJC5 mutations as cause of adult neuronal ceroid-lipofuscinosis. PLoS One 6, e26741.

Borghi, R., Marchese, R., Negro, A., Marinelli, L., Forloni, G., Zaccheo, D., Abbruzzese, G., and Tabaton, M. (2000). Full length alpha-synuclein is present in cerebrospinal fluid from Parkinson's disease and normal subjects. Neurosci Lett 287, 65-67.

Braak, H., Del Tredici, K., Bratzke, H., Hamm-Clement, J., Sandmann-Keil, D., and Rüb, U. (2002). Staging of the intracerebral inclusion body pathology associated with idiopathic Parkinson's disease (preclinical and clinical stages). J Neurol 249 Supp/ 3, III/1-5.

Braak, H., Del Tredici, K., Rüb, U., de Vos, R.A.I., Jansen Steur, E.N.H., and Braak, E. (2003). Staging of brain pathology related to sporadic Parkinson's disease. Neurobiology of aging 24, 197-211.

Buell, A.K., Galvagnion, C., Gaspar, R., Sparr, E., Vendruscolo, M., Knowles, T.P., Linse, S., and Dobson, C.M. (2014). Solution conditions determine the relative importance of nucleation and growth processes in a-synuclein aggregation. Proc Natl Acad Sci U S A 111, 7671-7676.

Burré, J., Sharma, M., and Südhof, T.C. (2015). Definition of a Molecular Pathway Mediating a-Synuclein Neurotoxicity. J Neurosci 35, 5221-5232.

Burré, J., Sharma, M., Tsetsenis, T., Buchman, V., Etherton, M.R., and Südhof, T.C. (2010). Alpha-synuclein promotes SNARE-complex assembly in vivo and in vitro. Science 329, 1663-1667.

Cadieux-Dion, M., Andermann, E., Lachance-Touchette, P., Ansorge, O., Meloche, C., Barnabé, A., Kuzniecky, R.I., Andermann, F., Faught, E., Leonberg, S., et al. (2013). Recurrent mutations in DNAJC5 cause autosomal dominant Kufs disease. Clin Genet 83, 571-575. 
Chandra, S., Gallardo, G., Fernández-Chacón, R., Schlüter, O.M., and Südhof, T.C. (2005). Alpha-synuclein cooperates with CSPalpha in preventing neurodegeneration. Cell 123, 383-396.

Chatterjee, D., and Kordower, J.H. (2019). Immunotherapy in Parkinson's disease: Current status and future directions. Neurobiol Dis 132, 104587.

Danzer, K.M., Kranich, L.R., Ruf, W.P., Cagsal-Getkin, O., Winslow, A.R., Zhu, L., Vanderburg, C.R., and McLean, P.J. (2012). Exosomal cell-to-cell transmission of alpha synuclein oligomers. Molecular neurodegeneration 7, 42.

Desplats, P., Lee, H.-J., Bae, E.-J., Patrick, C., Rockenstein, E., Crews, L., Spencer, B., Masliah, E., and Lee, S.-J. (2009). Inclusion formation and neuronal cell death through neuron-to-neuron transmission of alpha-synuclein. Proceedings of the National Academy of Sciences of the United States of America 106, 13010-13015.

Dull, T., Zufferey, R., Kelly, M., Mandel, R.J., Nguyen, M., Trono, D., and Naldini, L. (1998). A third-generation lentivirus vector with a conditional packaging system. Journal of virology $72,8463-8471$.

Ejlerskov, P., Rasmussen, I., Nielsen, T.T., Bergstrom, A.L., Tohyama, Y., Jensen, P.H., and Vilhardt, F. (2013). Tubulin polymerization-promoting protein (TPPP/p25alpha) promotes unconventional secretion of alpha-synuclein through exophagy by impairing autophagosome-lysosome fusion. J Biol Chem 288, 17313-17335.

El-Agnaf, O.M.A., Salem, S.A., Paleologou, K.E., Cooper, L.J., Fullwood, N.J., Gibson, M.J., Curran, M.D., Court, J.A., Mann, D.M.A., Ikeda, S.-i., et al. (2003). Alpha-synuclein implicated in Parkinson's disease is present in extracellular biological fluids, including human plasma. FASEB journal : official publication of the Federation of American Societies for Experimental Biology 17, 1945-1947.

Emmanouilidou, E., Melachroinou, K., Roumeliotis, T., Garbis, S.D., Ntzouni, M., Margaritis, L.H., Stefanis, L., and Vekrellis, K. (2010). Cell-produced alpha-synuclein is secreted in a calcium-dependent manner by exosomes and impacts neuronal survival. J Neurosci 30, 6838-6851.

Fernández-Chacón, R., Wölfel, M., Nishimune, H., Tabares, L., Schmitz, F., Castellano-Muñoz, M., Rosenmund, C., Montesinos, M.L., Sanes, J.R., Schneggenburger, R., et al. (2004). The synaptic vesicle protein CSP alpha prevents presynaptic degeneration. Neuron 42, 237-251.

Foulds, P.G., Diggle, P., Mitchell, J.D., Parker, A., Hasegawa, M., Masuda-Suzukake, M., Mann, D.M., and Allsop, D. (2013). A longitudinal study on a-synuclein in blood plasma as a biomarker for Parkinson's disease. Scientific reports 3, 2540.

Graham, J.M. (2001). Isolation of lysosomes from tissues and cells by differential and density gradient centrifugation. Current protocols in cell biology Chapter 3, Unit 3.6.

Huang, X., Wheeler, M.B., Kang, Y.H., Sheu, L., Lukacs, G.L., Trimble, W.S., and Gaisano, H.Y. (1998). Truncated SNAP25 (1-197), like botulinum neurotoxin A, can inhibit insulin secretion from HIT-T15 insulinoma cells. Molecular endocrinology (Baltimore, Md) 12, 1060-1070.

Ikegaki, N., and Kennett, R.H. (1989). Glutaraldehyde fixation of the primary antibody-antigen complex on nitrocellulose paper increases the overall sensitivity of immunoblot assay. Journal of immunological methods 124, 205-210. 
Imler, E., Pyon, J.S., Kindelay, S., Torvund, M., Zhang, Y.Q., Chandra, S.S., and Zinsmaier, K.E. (2019). A Drosophila model of neuronal ceroid lipofuscinosis CLN4 reveals a hypermorphic gain of function mechanism. Elife 8 .

Jang, A., Lee, H.J., Suk, J.E., Jung, J.W., Kim, K.P., and Lee, S.J. (2010). Non-classical exocytosis of alpha-synuclein is sensitive to folding states and promoted under stress conditions. J Neurochem 113, 1263-1274.

Kordower, J.H., Chu, Y., Hauser, R.A., Freeman, T.B., and Olanow, C.W. (2008). Lewy body-like pathology in long-term embryonic nigral transplants in Parkinson's disease. Nature medicine 14, 504-506.

Lee, H.J., Patel, S., and Lee, S.J. (2005). Intravesicular localization and exocytosis of alpha-synuclein and its aggregates. J Neurosci 25, 6016-6024.

Lee, H.J., Suk, J.E., Bae, E.J., and Lee, S.J. (2008). Clearance and deposition of extracellular alpha-synuclein aggregates in microglia. Biochem Biophys Res Commun 372, 423-428.

Lesuisse, C., and Martin, L.J. (2002). Long-term culture of mouse cortical neurons as a model for neuronal development, aging, and death. Journal of neurobiology 51, 9-23.

Li, J.-Y., Englund, E., Holton, J.L., Soulet, D., Hagell, P., Lees, A.J., Lashley, T., Quinn, N.P., Rehncrona, S., Björklund, A., et al. (2008). Lewy bodies in grafted neurons in subjects with Parkinson's disease suggest host-to-graft disease propagation. Nature medicine 14, 501-503.

Lim, N.K., Moestrup, V., Zhang, X., Wang, W.A., Møller, A., and Huang, F.D. (2018). An Improved Method for Collection of Cerebrospinal Fluid from Anesthetized Mice. Journal of visualized experiments : JoVE.

Lois, C., Hong, E.J., Pease, S., Brown, E.J., and Baltimore, D. (2002). Germline transmission and tissue-specific expression of transgenes delivered by lentiviral vectors. Science $295,868-872$.

Loria, F., Vargas, J.Y., Bousset, L., Syan, S., Salles, A., Melki, R., and Zurzolo, C. (2017). a-Synuclein transfer between neurons and astrocytes indicates that astrocytes play a role in degradation rather than in spreading. Acta Neuropathol 134, 789-808.

Luk, K.C., Kehm, V., Carroll, J., Zhang, B., O'Brien, P., Trojanowski, J.Q., and Lee, V.M. (2012). Pathological $\alpha$-synuclein transmission initiates Parkinson-like neurodegeneration in nontransgenic mice. Science 338, 949-953.

Luk, K.C., Song, C., O'Brien, P., Stieber, A., Branch, J.R., Brunden, K.R., Trojanowski, J.Q., and Lee, V.M. (2009). Exogenous alpha-synuclein fibrils seed the formation of Lewy body-like intracellular inclusions in cultured cells. Proc Natl Acad Sci U S A 106, 20051-20056.

Malik, B.R., Maddison, D.C., Smith, G.A., and Peters, O.M. (2019). Autophagic and endo-lysosomal dysfunction in neurodegenerative disease. Mol Brain 12, 100.

Martinez-Arca, S., Alberts, P., Zahraoui, A., Louvard, D., and Galli, T. (2000). Role of tetanus neurotoxin insensitive vesicleassociated membrane protein (TI-VAMP) in vesicular transport mediating neurite outgrowth. J Cell Biol 149, 889-900. 
Minakaki, G., Menges, S., Kittel, A., Emmanouilidou, E., Schaeffner, I., Barkovits, K., Bergmann, A., Rockenstein, E., Adame, A., Marxreiter, F., et al. (2018). Autophagy inhibition promotes SNCA/alpha-synuclein release and transfer via extracellular vesicles with a hybrid autophagosome-exosome-like phenotype. Autophagy 14, 98-119.

Naseri, N.N., Ergel, B., Kharel, P., Na, Y., Huang, Q., Huang, R., Dolzhanskaya, N., Burré, J., Velinov, M.T., and Sharma, M. (2020). Aggregation of mutant cysteine string protein- $\alpha$ via Fe-S cluster binding is mitigated by iron chelators. Nat Struct Mol Biol 27, 192-201.

Nosková, L., Stránecký, V., Hartmannová, H., Přistoupilová, A., Barešová, V., Ivánek, R., Hůlková, H., Jahnová, H., van der Zee, J., Staropoli, J.F., et al. (2011). Mutations in DNAJC5, encoding cysteine-string protein alpha, cause autosomaldominant adult-onset neuronal ceroid lipofuscinosis. Am J Hum Genet 89, 241-252.

Ogawa, N., Hirose, Y., Ohara, S., Ono, T., and Watanabe, Y. (1985). A simple quantitative bradykinesia test in MPTPtreated mice. Research communications in chemical pathology and pharmacology 50, 435-441.

Paumier, K.L., Luk, K.C., Manfredsson, F.P., Kanaan, N.M., Lipton, J.W., Collier, T.J., Steece-Collier, K., Kemp, C.J., Celano, S., Schulz, E., et al. (2015). Intrastriatal injection of pre-formed mouse a-synuclein fibrils into rats triggers asynuclein pathology and bilateral nigrostriatal degeneration. Neurobiol Dis 82, 185-199.

Peng, C., Trojanowski, J.Q., and Lee, V.M. (2020). Protein transmission in neurodegenerative disease. Nat Rev Neurol.

Rao, S.K., Huynh, C., Proux-Gillardeaux, V., Galli, T., and Andrews, N.W. (2004). Identification of SNAREs involved in synaptotagmin VII-regulated lysosomal exocytosis. J Biol Chem 279, 20471-20479.

Rasmussen, M.K., Mestre, H., and Nedergaard, M. (2018). The glymphatic pathway in neurological disorders. Lancet Neurol $17,1016-1024$.

Rey, N.L., George, S., Steiner, J.A., Madaj, Z., Luk, K.C., Trojanowski, J.Q., Lee, V.M., and Brundin, P. (2018). Spread of aggregates after olfactory bulb injection of $\alpha$-synuclein fibrils is associated with early neuronal loss and is reduced long term. Acta Neuropathol 135, 65-83.

Rey, N.L., Petit, G.H., Bousset, L., Melki, R., and Brundin, P. (2013). Transfer of human a-synuclein from the olfactory bulb to interconnected brain regions in mice. Acta Neuropathologica 126, 555-573.

Reyes, J.F., Rey, N.L., Bousset, L., Melki, R., Brundin, P., and Angot, E. (2014). Alpha-synuclein transfers from neurons to oligodendrocytes. Glia 62, 387-398.

Smith, A.J., and Verkman, A.S. (2018). The "glymphatic" mechanism for solute clearance in Alzheimer's disease: game changer or unproven speculation? Faseb j 32, 543-551.

Spillantini, M.G., Crowther, R.A., Jakes, R., Hasegawa, M., and Goedert, M. (1998). alpha-Synuclein in filamentous inclusions of Lewy bodies from Parkinson's disease and dementia with lewy bodies. Proceedings of the National Academy of Sciences of the United States of America 95, 6469-6473. 
Spillantini, M.G., Schmidt, M.L., Lee, V.M., Trojanowski, J.Q., Jakes, R., and Goedert, M. (1997). Alpha-synuclein in Lewy bodies. Nature 388, 839-840.

Sung, J.Y., Park, S.M., Lee, C.H., Um, J.W., Lee, H.J., Kim, J., Oh, Y.J., Lee, S.T., Paik, S.R., and Chung, K.C. (2005). Proteolytic cleavage of extracellular secreted \{alpha\}-synuclein via matrix metalloproteinases. J Biol Chem 280, 2521625224.

Velinov, M., Dolzhanskaya, N., Gonzalez, M., Powell, E., Konidari, I., Hulme, W., Staropoli, J.F., Xin, W., Wen, G.Y., Barone, R., et al. (2012). Mutations in the gene DNAJC5 cause autosomal dominant Kufs disease in a proportion of cases: study of the Parry family and 8 other families. PLoS One 7, e29729.

Volpicelli-Daley, L.A., Luk, K.C., Patel, T.P., Tanik, S.A., Riddle, D.M., Stieber, A., Meaney, D.F., Trojanowski, J.Q., and Lee, V.M. (2011a). Exogenous $\alpha$-synuclein fibrils induce Lewy body pathology leading to synaptic dysfunction and neuron death. Neuron $72,57-71$.

Volpicelli-Daley, L.A., Luk, K.C., Patel, T.P., Tanik, S.A., Riddle, D.M., Stieber, A., Meaney, D.F., Trojanowski, J.Q., and Lee, V.M.Y. (2011b). Exogenous a-synuclein fibrils induce Lewy body pathology leading to synaptic dysfunction and neuron death. Neuron $72,57-71$.

Yamada, K., and Iwatsubo, T. (2018). Extracellular a-synuclein levels are regulated by neuronal activity. Mol Neurodegener $13,9$.

Yamamoto, A., and Simonsen, A. (2011). The elimination of accumulated and aggregated proteins: a role for aggrephagy in neurodegeneration. Neurobiol Dis 43, 17-28.

Yee, J.K., Miyanohara, A., LaPorte, P., Bouic, K., Burns, J.C., and Friedmann, T. (1994). A general method for the generation of high-titer, pantropic retroviral vectors: highly efficient infection of primary hepatocytes. Proc Natl Acad Sci $U$ S A 91, 9564-9568. 